\title{
Modeling the Stereoselectivity of the Johnson-Claisen Rearrangements in the Danishefsky Synthesis of Gelsemine
}

\author{
C. Ozturk and V. Aviyente* \\ Chemistry Department, Bogazici University 34342, Bebek, Istanbul, Turkey \\ K. N. Houk \\ Department of Chemistry and Biochemistry, University of California, Los Angeles, \\ California 90095-1569 \\ aviye@boun.edu.tr
}

Supporting Information

Table of Contents

*Data of TS-3E-4in:

S5-S6

Table S1: Cartesian coordinates, energy and imaginary frequency of B3LYP/6-31G* optimization of transition state from compound $3 \mathrm{E}$ to $4 \mathrm{in}$.

* Data of TS-3E-4out:

S6-S7

Table S2: Cartesian coordinates, energy and imaginary frequency of B3LYP/6-31G* optimization of transition state from compound $3 \mathrm{E}$ to 4 out.

* Data of TS-3Z-4in:

S8-S9

Table S3 :Cartesian coordinates, energy and imaginary frequency of B3LYP/6-31G* optimization of transition state from compound $3 \mathrm{Z}$ to $4 \mathrm{in}$.

* Data of TS-3Z-4out:

Table S4:Cartesian coordinates, energy and imaginary frequency of B3LYP/6-31G* optimization of transition state from compound $3 \mathrm{Z}$ to 4 out. 
Table S5: Cartesian coordinates, energy and imaginary frequency of B3LYP/6-31G* optimization of transition state from compound $5 \mathrm{E}$ to $6 \mathrm{in}$.

* Data of TS-5E-6out:

Table S6: Cartesian coordinates, energy and imaginary frequency of B3LYP/6-31G* optimization of transition state from compound $5 \mathrm{E}$ to 6out.

* Data of TS-5Z-6in:

Table S7: Cartesian coordinates, energy and imaginary frequency of B3LYP/6-31G* optimization of transition state from compound $5 \mathrm{Z}$ to 6in.

* Data of TS-5Z-6out:

Table S8: Cartesian coordinates, energy and imaginary frequency of B3LYP/6-31G* optimization of transition state from compound $5 \mathrm{Z}$ to 6out.

* Data of TS-7E-8in:

Table S9: Cartesian coordinates, energy and imaginary frequency of B3LYP/6-31G* optimization of transition state from compound 7E to 8in.

Table S10: Cartesian coordinates, energy and imaginary frequency of B3LYP/6-31+G** optimization of transition state from compound 7E to 8in.

Table S11: Cartesian coordinates and energy of MPW1K/6-31+G** optimization of transition state from compound 7E to 8in.

Table S12: Energetics of IEFPCM/B3LYP/6-31+G**//B3LYP/6-31+G** ( $\varepsilon=$ toluene) single point calculation of transition state from compound $7 \mathrm{E}$ to 8 in.

Table S13: Energetics of IEFPCM/B3LYP/6-31+G**//B3LYP/6-31+G** ( $E=$ water) single point calculation of transition state from compound $7 \mathrm{E}$ to 8 in.

S21

Table S14: Energetics of IEFPCM/ MPW1K/6-31+G**//MPW1K/6-31+G** ( $\varepsilon=$ =toluene) single point calculation of transition state from compound $7 \mathrm{E}$ to 8 in.

Table S15 Energetics of IEFPCM/ MPW1K/6-31+G**//MPW1K/6-31+G** ( $\mathcal{E}=$ water) single point calculation of transition state from compound $7 \mathrm{E}$ to 8in. 
* Data of TS-7E-8out:

Table S16 Cartesian coordinates, energy and imaginary frequency of B3LYP/6-31G* optimization of transition state from compound 7E to 8out.

Table S17 Cartesian coordinates and energy of B3LYP/6-31+G** optimization of transition state from compound 7E to 8out.

Table S18 Cartesian coordinates and energy of MPW1K/6-31+G** optimization of transition state from compound 7E to 8out.

Table S19 Energetics of IEFPCM/B3LYP/6-31+G**//B3LYP/6-31+G** ( $E=$ toluene) single point calculation of transition state from compound $7 \mathrm{E}$ to 8out.

Table S20 Energetics of IEFPCM/B3LYP/6-31+G**//B3LYP/6-31+G** ( $\varepsilon=$ water) single point calculation of transition state from compound $7 \mathrm{E}$ to 8out.

Table S21 Energetics of IEFPCM/ MPW1K/6-31+G**//MPW1K/6-31+G** ( $\mathcal{E}=$ toluene) single point calculation of transition state from compound $7 \mathrm{E}$ to 8out.

Table S22 Energetics of IEFPCM/ MPW1K/6-31+G**//MPW1K/6-31+G** ( $\varepsilon=$ =water) single point calculation of transition state from compound $7 \mathrm{E}$ to 8out.

* Data of TS-7Z-8in:

Table S23 Cartesian coordinates, energy and imaginary frequency of B3LYP/6-31G* optimization of transition state from compound $7 \mathrm{Z}$ to 8 in.

Table S24 Cartesian coordinates and energy of B3LYP/6-31+G** optimization of transition state from compound $7 \mathrm{Z}$ to 8 in.

Table S25 Cartesian coordinates and energy of MPW1K/6-31+G** optimization of transition state from compound $7 \mathrm{Z}$ to 8 in.

Table S26 Energetics of IEFPCM/B3LYP/6-31+G**//B3LYP/6-31+G** ( $\varepsilon=$ toluene) single point calculation of transition state from compound $7 \mathrm{Z}$ to $8 \mathrm{in}$.

Table S27 Energetics of IEFPCM/B3LYP/6-31+G**//B3LYP/6-31+G** ( $\varepsilon=$ =water) single point calculation of transition state from compound $7 \mathrm{Z}$ to $8 \mathrm{in}$.

Table S28 Energetics of IEFPCM/ MPW1K/6-31+G**//MPW1K/6-31+G** ( $\varepsilon=$ toluene) single point calculation of transition state from compound $7 \mathrm{Z}$ to 8 in. 
Table S29 Energetics of IEFPCM/ MPW1K/6-31+G**//MPW1K/6-31+G** ( $E=$ water) single point calculation of transition state from compound $7 \mathrm{Z}$ to $8 \mathrm{in}$.

* Data of TS-7Z-8out:

Table S30 Cartesian coordinates, energy and imaginary frequency of B3LYP/6-31G* optimization of transition state from compound $7 \mathrm{Z}$ to 8out.

Table S31 Cartesian coordinates and energy of B3LYP/6-31+G** optimization of transition state from compound $7 \mathrm{Z}$ to 8out.

Table S32 Cartesian coordinates and energy of MPW1K/6-31+G** optimization of transition state from compound $7 \mathrm{Z}$ to 8out.

Table S33 Energetics of IEFPCM/B3LYP/6-31+G**//B3LYP/6-31+G** ( $\varepsilon=$ toluene) single point calculation of transition state from compound $7 \mathrm{Z}$ to 8 out.

Table S34 Energetics of IEFPCM/B3LYP/6-31+G**//B3LYP/6-31+G** ( $E=$ water) single point calculation of transition state from compound $7 \mathrm{Z}$ to 8 out.

Table S35 Energetics of IEFPCM/ MPW1K/6-31+G**//MPW1K/6-31+G** ( $\varepsilon=$ toluene) single point calculation of transition state from compound $7 \mathrm{Z}$ to 8out.

Table S36 Energetics of IEFPCM/ MPW1K/6-31+G**//MPW1K/6-31+G** ( $E=$ =water) single point calculation of transition state from compound $7 \mathrm{Z}$ to 8 out. 
Table S1 Cartesian coordinates, energy and imaginary frequency of B3LYP/6-31G* optimization of transition state from compound $3 \mathrm{E}$ to $4 \mathrm{in}$.

$\mathrm{W}=-422.8167$

Standard orientation:

\begin{tabular}{|c|c|c|c|c|c|}
\hline Center & Atomic & & omic & Coordinates & (Angstrom \\
\hline Number & Number & & Type & $X$ & $\mathrm{Z}$ \\
\hline 1 & 6 & 0 & -1.734782 & 1.405978 & -0.069404 \\
\hline 2 & 6 & 0 & -1.395403 & 0.184605 & 0.787288 \\
\hline 3 & 1 & 0 & -4.108654 & 0.485680 & 1.261849 \\
\hline 4 & 6 & 0 & 0.124772 & 0.281648 & 0.931039 \\
\hline 5 & 6 & 0 & -1.016703 & 1.334789 & -1.405292 \\
\hline 6 & 6 & 0 & -1.095855 & 2.481594 & 0.865015 \\
\hline 7 & 1 & 0 & -2.804167 & 1.558306 & -0.221870 \\
\hline 8 & 6 & 0 & -0.107909 & 3.519435 & 0.302401 \\
\hline 9 & 1 & 0 & -0.125896 & 3.695169 & -0.778287 \\
\hline 10 & 1 & 0 & -1.811298 & 2.845116 & 1.606223 \\
\hline 11 & 1 & 0 & 0.508177 & -0.394738 & 1.694753 \\
\hline 12 & 6 & 0 & 0.182221 & 1.773514 & 1.400931 \\
\hline 13 & 6 & 0 & 0.815629 & -0.053327 & -0.425038 \\
\hline 14 & 1 & 0 & 0.357547 & 1.824571 & 2.482056 \\
\hline 15 & 8 & 0 & 1.031061 & 2.716749 & 0.699862 \\
\hline 16 & 1 & 0 & -0.118193 & 4.480768 & 0.831945 \\
\hline 17 & 6 & 0 & 2.343072 & 0.023632 & -0.408333 \\
\hline 18 & 6 & 0 & 0.164283 & 0.726143 & -1.549656 \\
\hline 19 & 1 & 0 & 0.568233 & -1.105773 & -0.599024 \\
\hline 20 & 1 & 0 & 0.647531 & 0.694379 & -2.524791 \\
\hline 21 & 1 & 0 & -1.489760 & 1.822566 & -2.254287 \\
\hline 22 & 1 & 0 & -1.559821 & -2.429143 & -0.038234 \\
\hline 23 & 1 & 0 & -1.698064 & -0.984961 & 2.526119 \\
\hline 24 & 1 & 0 & -5.699456 & -0.010597 & -0.628234 \\
\hline 25 & 1 & 0 & -5.831647 & -1.550924 & -1.503382 \\
\hline 26 & 8 & 0 & -4.014362 & -1.673772 & 0.505841 \\
\hline 27 & 6 & 0 & -2.019850 & -1.738307 & -0.730456 \\
\hline 28 & 1 & 0 & -4.132309 & -0.745810 & 2.608582 \\
\hline 29 & 1 & 0 & -5.643005 & -0.021884 & -2.414285 \\
\hline 30 & 6 & 0 & -5.357313 & -0.563047 & -1.509554 \\
\hline 31 & 6 & 0 & -3.357534 & -1.437620 & -0.566284 \\
\hline 32 & 8 & 0 & -3.936082 & -0.671400 & -1.558638 \\
\hline 33 & 6 & 0 & -2.180903 & -0.313792 & 1.816991 \\
\hline 34 & 1 & 0 & -1.542048 & -1.562404 & -1.685777 \\
\hline 35 & 6 & 0 & -3.580404 & -0.302703 & 1.787561 \\
\hline
\end{tabular}




$\begin{array}{rrrrrr}36 & 6 & 0 & 5.187133 & 0.217906 & -0.530239 \\ 37 & 6 & 0 & 2.993984 & 1.181501 & -0.863104 \\ 38 & 6 & 0 & 3.191030 & -1.045241 & -0.038334 \\ 39 & 6 & 0 & 4.585961 & -0.957256 & -0.102163 \\ 40 & 6 & 0 & 4.381141 & 1.289350 & -0.916578 \\ 41 & 1 & 0 & 2.382155 & 2.023762 & -1.155878 \\ 42 & 7 & 0 & 2.677739 & -2.350201 & 0.419435 \\ 43 & 1 & 0 & 5.173003 & -1.820534 & 0.184662 \\ 44 & 1 & 0 & 4.831494 & 2.215601 & -1.262571 \\ 45 & 1 & 0 & 6.269719 & 0.292372 & -0.566653 \\ 46 & 8 & 0 & 3.406244 & -3.331419 & 0.274237 \\ 47 & 8 & 0 & 1.556498 & -2.404273 & 0.933735 \\ ----------------------------------------------------------------\end{array}$

\section{$\mathrm{HF}=-1205.367891$}

Sum of electronic and zero-point Energies $=\quad-1204.988365$

Sum of electronic and thermal Energies= $\quad-1204.966263$

Sum of electronic and thermal Enthalpies $=\quad-1204.965319$

Sum of electronic and thermal Free Energies $=\quad-1205.039733$

Table S2 Cartesian coordinates, energy and imaginary frequency of B3LYP/6-31G* optimization of transition state from compound $3 \mathrm{E}$ to 4out.

Standard orientation:

\begin{tabular}{|c|c|c|c|c|}
\hline \multirow{2}{*}{$\begin{array}{l}\text { Center } \\
\text { Number }\end{array}$} & \multirow{2}{*}{$\begin{array}{l}\text { Atomic } \\
\text { Number }\end{array}$} & \multirow{2}{*}{$\begin{array}{r}\text { Atomic } \\
\text { Type }\end{array}$} & \multicolumn{2}{|c|}{ Coordinates (Angstroms) } \\
\hline & & & $\mathrm{X}$ & $\mathrm{Z}$ \\
\hline 1 & 6 & -1.666202 & -0.960417 & 0.824229 \\
\hline 2 & 6 & -1.258618 & 0.398126 & 0.265033 \\
\hline 3 & 1 & -3.136038 & 2.889985 & 1.636467 \\
\hline 4 & 6 & 0.057661 & 0.096872 & -0.436158 \\
\hline 5 & 6 & -0.646748 & -1.254655 & 1.925341 \\
\hline 6 & 6 & -1.468119 & -1.888003 & -0.404555 \\
\hline 7 & 1 & -2.684869 & -1.007872 & 1.212092 \\
\hline 8 & 6 & -0.567478 & -3.131227 & -0.299727 \\
\hline 9 & 1 & -0.357336 & -3.525425 & 0.700636 \\
\hline 10 & 1 & -2.400054 & -2.011488 & -0.956568 \\
\hline 11 & 1 & 0.356372 & 0.874488 & -1.138349 \\
\hline 12 & 6 & -0.260578 & -1.252184 & -1.141029 \\
\hline 13 & 6 & 1.121535 & 0.054180 & 0.724629 \\
\hline 14 & 1 & -0.350892 & -1.115576 & -2.224668 \\
\hline 15 & 8 & 0.564831 & -2.411203 & -0.845620 \\
\hline
\end{tabular}




\begin{tabular}{|c|c|c|c|c|c|}
\hline 16 & 1 & 0 & -0.868313 & -3.954033 & -0.960915 \\
\hline 17 & 6 & 0 & 2.560297 & -0.215043 & 0.286785 \\
\hline 18 & 6 & 0 & 0.623630 & -0.841688 & 1.843761 \\
\hline 19 & 1 & 0 & 1.109302 & 1.074792 & 1.124441 \\
\hline 20 & 1 & 0 & 1.323607 & -1.070334 & 2.645519 \\
\hline 21 & 1 & 0 & -0.982209 & -1.831698 & 2.784909 \\
\hline 22 & 1 & 0 & -2.814727 & -0.222261 & -2.392759 \\
\hline 23 & 1 & 0 & -1.096309 & 2.507482 & 0.321682 \\
\hline 24 & 1 & 0 & -6.521216 & 0.529518 & -0.346377 \\
\hline 25 & 1 & 0 & -5.659716 & 0.151984 & 1.160534 \\
\hline 26 & 8 & 0 & -4.148679 & 1.829157 & -0.161819 \\
\hline 27 & 6 & 0 & -2.888992 & 0.653627 & -1.759832 \\
\hline 28 & 1 & 0 & -3.360916 & 1.094587 & 1.893346 \\
\hline 29 & 1 & 0 & -6.331647 & -1.174498 & 0.167917 \\
\hline 30 & 6 & 0 & -5.855144 & -0.192083 & 0.139761 \\
\hline 31 & 6 & 0 & -3.928689 & 0.784287 & -0.860582 \\
\hline 32 & 8 & 0 & -4.651876 & -0.368560 & -0.605979 \\
\hline 33 & 6 & 0 & -1.643132 & 1.649534 & 0.709684 \\
\hline 34 & 1 & 0 & -2.353114 & 1.545113 & -2.053924 \\
\hline 35 & 6 & 0 & -2.868891 & 1.883636 & 1.336129 \\
\hline 36 & 6 & 0 & 5.267601 & -0.797667 & -0.396700 \\
\hline 37 & 6 & 0 & 3.083448 & -1.516131 & 0.353428 \\
\hline 38 & 6 & 0 & 3.470168 & 0.787853 & -0.119375 \\
\hline 39 & 6 & 0 & 4.800567 & 0.508052 & -0.449705 \\
\hline 40 & 6 & 0 & 4.400826 & -1.811481 & 0.013119 \\
\hline 41 & 1 & 0 & 2.418902 & -2.313748 & 0.655933 \\
\hline 42 & 7 & 0 & 3.102213 & 2.213612 & -0.204095 \\
\hline 43 & 1 & 0 & 5.445230 & 1.327728 & -0.740690 \\
\hline 44 & 1 & 0 & 4.748707 & -2.839222 & 0.068686 \\
\hline 45 & 1 & 0 & 6.295787 & -1.018078 & -0.666993 \\
\hline 46 & 8 & 0 & 1.917698 & 2.515623 & -0.380413 \\
\hline 47 & 8 & 0 & 4.006164 & 3.042452 & -0.104628 \\
\hline
\end{tabular}

$\mathrm{W}=-372.7736$

$\mathrm{HF}=-1205.3727095$

Sum of electronic and zero-point Energies $=\quad-1204.993437$

Sum of electronic and thermal Energies $=\quad-1204.971229$

Sum of electronic and thermal Enthalpies $=\quad-1204.970285$

Sum of electronic and thermal Free Energies $=\quad-1205.045169$ 
Table S3 Cartesian coordinates, energy and imaginary frequency of B3LYP/6-31G* optimization of transition state from compound $3 \mathrm{Z}$ to $4 \mathrm{in}$.

Standard orientation:

\begin{tabular}{|c|c|c|c|c|c|}
\hline \multirow{2}{*}{$\begin{array}{l}\text { Center } \\
\text { Number }\end{array}$} & \multirow{2}{*}{\multicolumn{2}{|c|}{$\begin{array}{l}\text { Atomic } \\
\text { Number }\end{array}$}} & \multirow{2}{*}{$\begin{array}{l}\text { Atomic } \\
\text { Type }\end{array}$} & \multicolumn{2}{|c|}{ Coordinates (Angstroms } \\
\hline & & & & $\mathrm{X}$ & $\mathrm{Z}$ \\
\hline 1 & 6 & 0 & 1.567087 & 2.470788 & 0.554348 \\
\hline 2 & 6 & 0 & 1.828032 & 1.144951 & -0.153278 \\
\hline 3 & 1 & 0 & 4.194580 & -0.456661 & -2.021180 \\
\hline 4 & 6 & 0 & 0.431236 & 0.730075 & -0.631180 \\
\hline 5 & 6 & 0 & 0.619626 & 2.253140 & 1.720975 \\
\hline 6 & 6 & 0 & 0.876537 & 3.209363 & -0.634144 \\
\hline 7 & 1 & 0 & 2.484053 & 2.969898 & 0.880519 \\
\hline 8 & 6 & 0 & -0.489337 & 3.894313 & -0.446711 \\
\hline 9 & 1 & 0 & -0.792743 & 4.133277 & 0.578193 \\
\hline 10 & 1 & 0 & 1.604033 & 3.740771 & -1.252139 \\
\hline 11 & 1 & 0 & 0.449236 & -0.080753 & -1.354054 \\
\hline 12 & 6 & 0 & 0.051854 & 2.083115 & -1.319717 \\
\hline 13 & 6 & 0 & -0.454166 & 0.295246 & 0.575135 \\
\hline 14 & 1 & 0 & 0.145618 & 1.995689 & -2.408493 \\
\hline 15 & 8 & 0 & -1.191328 & 2.736555 & -0.961738 \\
\hline 16 & 1 & 0 & -0.644007 & 4.770026 & -1.089961 \\
\hline 17 & 6 & 0 & -1.922306 & -0.035198 & 0.270586 \\
\hline 18 & 6 & 0 & -0.307618 & 1.290380 & 1.711255 \\
\hline 19 & 1 & 0 & -0.000932 & -0.644037 & 0.918995 \\
\hline 20 & 1 & 0 & -0.958839 & 1.155305 & 2.573201 \\
\hline 21 & 1 & 0 & 0.710999 & 2.917206 & 2.577925 \\
\hline 22 & 1 & 0 & 1.841858 & -0.446714 & 2.484618 \\
\hline 23 & 1 & 0 & 3.863105 & 1.519121 & -0.613653 \\
\hline 24 & 1 & 0 & 2.725360 & -4.164171 & 0.399048 \\
\hline 25 & 1 & 0 & 2.168555 & -3.278059 & -1.043507 \\
\hline 26 & 8 & 0 & 3.674529 & -1.511477 & -0.059057 \\
\hline 27 & 6 & 0 & 2.601081 & -0.413054 & 1.713613 \\
\hline 28 & 1 & 0 & 2.425123 & -0.900535 & -1.902212 \\
\hline 29 & 1 & 0 & 0.973337 & -4.074922 & 0.008240 \\
\hline 30 & 6 & 0 & 1.923022 & -3.541009 & -0.012594 \\
\hline 31 & 6 & 0 & 2.746573 & -1.459038 & 0.820520 \\
\hline 32 & 8 & 0 & 1.725185 & -2.378983 & 0.797561 \\
\hline 33 & 6 & 0 & 2.999926 & 0.897605 & -0.852708 \\
\hline 34 & 1 & 0 & 3.437076 & 0.257096 & 1.854798 \\
\hline 35 & 6 & 0 & 3.248303 & -0.310657 & -1.513519 \\
\hline 36 & 6 & 0 & -4.687558 & -0.699329 & 0.012017 \\
\hline 37 & 6 & 0 & -2.941225 & 0.892227 & 0.538230 \\
\hline
\end{tabular}




\begin{tabular}{|c|c|c|c|c|c|}
\hline 38 & 6 & 0 & -2.364996 & -1.309329 & -0.135480 \\
\hline 39 & 6 & 0 & -3.713140 & -1.655595 & -0.244821 \\
\hline 40 & 6 & 0 & -4.292533 & 0.580758 & 0.400564 \\
\hline 41 & 1 & 0 & -2.655107 & 1.886820 & 0.853355 \\
\hline 42 & 7 & 0 & -1.427259 & -2.385821 & -0.492620 \\
\hline 43 & 1 & 0 & -3.973113 & -2.666968 & -0.535381 \\
\hline 44 & 1 & 0 & -5.038960 & 1.342137 & 0.608807 \\
\hline 45 & 1 & 0 & -5.738494 & -0.953608 & -0.086390 \\
\hline 46 & 8 & 0 & -1.661854 & -3.514128 & -0.060767 \\
\hline 47 & 8 & 0 & -0.490571 & -2.111053 & -1.243615 \\
\hline
\end{tabular}

$\mathrm{W}=-408.9902$

$\mathrm{HF}=-1205.3676301$

Sum of electronic and zero-point Energies $=\quad-1204.987938$

Sum of electronic and thermal Energies $=\quad-1204.965946$

Sum of electronic and thermal Enthalpies $=\quad-1204.965002$

Sum of electronic and thermal Free Energies $=\quad-1205.038237$

Table S4 Cartesian coordinates, energy and imaginary frequency of B3LYP/6-31G* optimization of transition state from compound $3 \mathrm{Z}$ to 4 out.

Standard orientation:

\begin{tabular}{|c|c|c|c|c|c|}
\hline \multirow{2}{*}{$\begin{array}{l}\text { Center } \\
\text { Number }\end{array}$} & \multirow{2}{*}{\multicolumn{2}{|c|}{$\begin{array}{l}\text { Atomic } \\
\text { Number }\end{array}$}} & \multirow{2}{*}{$\begin{array}{r}\text { Atomic } \\
\text { Type }\end{array}$} & \multicolumn{2}{|c|}{ Coordinates (Angstroms) } \\
\hline & & & & $\mathrm{X}$ & $\mathrm{Z}$ \\
\hline 1 & 6 & 0 & 1.537117 & 2.312827 & 0.881034 \\
\hline 2 & 6 & 0 & 1.646970 & 0.795130 & 0.832617 \\
\hline 3 & 1 & 0 & 1.574401 & -1.933972 & 1.249756 \\
\hline 4 & 6 & 0 & 0.462053 & 0.397172 & -0.043256 \\
\hline 5 & 6 & 0 & 0.279782 & 2.592001 & 1.705544 \\
\hline 6 & 6 & 0 & 1.341407 & 2.674590 & -0.615249 \\
\hline 7 & 1 & 0 & 2.404384 & 2.802975 & 1.332612 \\
\hline 8 & 6 & 0 & 0.127298 & 3.509571 & -1.059093 \\
\hline 9 & 1 & 0 & -0.382653 & 4.105426 & -0.293911 \\
\hline 10 & 1 & 0 & 2.288752 & 2.913951 & -1.101147 \\
\hline 11 & 1 & 0 & 0.532783 & -0.607891 & -0.451680 \\
\hline 12 & 6 & 0 & 0.535833 & 1.470241 & -1.167487 \\
\hline 13 & 6 & 0 & -0.789238 & 0.466349 & 0.909578 \\
\hline 14 & 1 & 0 & 0.880405 & 1.021212 & -2.104442 \\
\hline 15 & 8 & 0 & -0.620649 & 2.320035 & -1.405031 \\
\hline 16 & 1 & 0 & 0.313471 & 4.132291 & -1.943631 \\
\hline
\end{tabular}




\begin{tabular}{|c|c|c|c|c|c|}
\hline 17 & 6 & 0 & -2.133637 & 0.133569 & 0.259485 \\
\hline 18 & 6 & 0 & -0.780887 & 1.776319 & 1.675992 \\
\hline 19 & 1 & 0 & -0.601387 & -0.320822 & 1.649615 \\
\hline 20 & 1 & 0 & -1.655781 & 2.001674 & 2.283538 \\
\hline 21 & 1 & 0 & 0.267783 & 3.486638 & 2.325059 \\
\hline 22 & 1 & 0 & 4.246374 & 0.824613 & -0.203664 \\
\hline 23 & 1 & 0 & 2.995130 & 0.543522 & 2.444205 \\
\hline 24 & 1 & 0 & 1.751124 & -3.317421 & -0.818548 \\
\hline 25 & 1 & 0 & 3.384347 & -3.514731 & -1.501799 \\
\hline 26 & 8 & 0 & 3.674287 & -1.689211 & 0.295710 \\
\hline 27 & 6 & 0 & 3.548857 & 0.344157 & -0.875393 \\
\hline 28 & 1 & 0 & 2.923236 & -1.902295 & 2.482942 \\
\hline 29 & 1 & 0 & 1.973107 & -3.322162 & -2.588691 \\
\hline 30 & 6 & 0 & 2.416247 & -3.022386 & -1.636451 \\
\hline 31 & 6 & 0 & 3.335286 & -1.015758 & -0.732631 \\
\hline 32 & 8 & 0 & 2.563405 & -1.602915 & -1.713625 \\
\hline 33 & 6 & 0 & 2.295900 & 0.029745 & 1.785630 \\
\hline 34 & 1 & 0 & 3.319867 & 0.832451 & -1.815069 \\
\hline 35 & 6 & 0 & 2.359858 & -1.364443 & 1.729161 \\
\hline 36 & 6 & 0 & -4.716122 & -0.363119 & -0.853994 \\
\hline 37 & 6 & 0 & -2.656765 & -1.172184 & 0.110511 \\
\hline 38 & 6 & 0 & -2.982473 & 1.171986 & -0.157286 \\
\hline 39 & 6 & 0 & -4.239260 & 0.940107 & -0.708903 \\
\hline 40 & 6 & 0 & -3.923748 & -1.419990 & -0.430523 \\
\hline 41 & 7 & 0 & -1.927120 & -2.380435 & 0.532764 \\
\hline 42 & 1 & 0 & -2.623272 & 2.187527 & -0.063718 \\
\hline 43 & 1 & 0 & -4.845902 & 1.783373 & -1.027076 \\
\hline 44 & 1 & 0 & -4.262871 & -2.445298 & -0.505505 \\
\hline 45 & 1 & 0 & -5.693191 & -0.555458 & -1.286496 \\
\hline 46 & 8 & 0 & -0.694753 & -2.334877 & 0.614708 \\
\hline 47 & 8 & 0 & -2.583180 & -3.391515 & 0.777311 \\
\hline
\end{tabular}

$\mathrm{W}=-376.7665$

$\mathrm{HF}=-1205.373998$

Sum of electronic and zero-point Energies $=\quad-1204.994514$

Sum of electronic and thermal Energies= $\quad-1204.972378$

Sum of electronic and thermal Enthalpies= $\quad-1204.971434$

Sum of electronic and thermal Free Energies $=\quad-1205.045914$ 
Table S5 Cartesian coordinates, energy and imaginary frequency of B3LYP/6-31G* optimization of transition state from compound $5 \mathrm{E}$ to $6 \mathrm{in}$.

Standard orientation:

\begin{tabular}{|c|c|c|c|c|c|}
\hline \multirow{2}{*}{$\begin{array}{l}\text { Center } \\
\text { Number }\end{array}$} & \multirow{2}{*}{\multicolumn{2}{|c|}{$\begin{array}{l}\text { Atomic } \\
\text { Number }\end{array}$}} & \multirow{2}{*}{$\begin{array}{r}\text { Atomic } \\
\text { Type }\end{array}$} & \multicolumn{2}{|c|}{ Coordinates (Angstroms } \\
\hline & & & & $\mathrm{X}$ & $\mathrm{Z}$ \\
\hline 1 & 6 & 0 & -4.130968 & -1.967432 & -0.477176 \\
\hline 2 & 6 & 0 & -5.082059 & -0.959566 & -0.308741 \\
\hline 3 & 6 & 0 & -4.657547 & 0.353505 & -0.152811 \\
\hline 4 & 6 & 0 & -3.290114 & 0.646061 & -0.140559 \\
\hline 5 & 6 & 0 & -2.301162 & -0.343115 & -0.315408 \\
\hline 6 & 6 & 0 & -2.773871 & -1.654321 & -0.491066 \\
\hline 7 & 6 & 0 & -0.795963 & -0.093004 & -0.353443 \\
\hline 8 & 6 & 0 & -0.140571 & -0.220444 & 1.071289 \\
\hline 9 & 6 & 0 & 1.383108 & -0.231763 & 0.955529 \\
\hline 10 & 6 & 0 & 1.632273 & -1.602323 & 0.322848 \\
\hline 11 & 6 & 0 & 1.038467 & -1.644394 & -1.081075 \\
\hline 12 & 6 & 0 & -0.094067 & -0.991340 & -1.359461 \\
\hline 13 & 6 & 0 & -0.313734 & -1.630268 & 1.638250 \\
\hline 14 & 6 & 0 & 0.690824 & -2.414552 & 1.228843 \\
\hline 15 & 6 & 0 & 2.201039 & 0.416128 & 1.865475 \\
\hline 16 & 6 & 0 & 3.592752 & 0.304715 & 1.862386 \\
\hline 17 & 8 & 0 & 4.132368 & 1.404065 & 0.337144 \\
\hline 18 & 6 & 0 & 3.430650 & 1.052393 & -0.670350 \\
\hline 19 & 8 & 0 & 3.916241 & 0.084453 & -1.530638 \\
\hline 20 & 6 & 0 & 5.321051 & -0.148226 & -1.467925 \\
\hline 21 & 7 & 0 & -2.954919 & 2.067880 & 0.058759 \\
\hline 22 & 8 & 0 & -3.790456 & 2.902764 & -0.285720 \\
\hline 23 & 6 & 0 & 2.121271 & 1.440279 & -0.872527 \\
\hline 24 & 8 & 0 & -1.870442 & 2.352225 & 0.573806 \\
\hline 25 & 1 & 0 & 4.069893 & -0.594020 & 1.487671 \\
\hline 26 & 1 & 0 & 2.671861 & -1.932595 & 0.332757 \\
\hline 27 & 1 & 0 & 0.815740 & -3.474798 & 1.427636 \\
\hline 28 & 1 & 0 & -0.523050 & 0.574017 & 1.714637 \\
\hline 29 & 1 & 0 & -1.160870 & -1.931878 & 2.245806 \\
\hline 30 & 1 & 0 & -0.628298 & 0.942926 & -0.661402 \\
\hline 31 & 1 & 0 & -0.531859 & -1.045684 & -2.354994 \\
\hline 32 & 1 & 0 & 1.544491 & -2.237530 & -1.838077 \\
\hline 33 & 1 & 0 & 1.735342 & 2.262074 & -0.285831 \\
\hline 34 & 1 & 0 & 1.759115 & 1.237103 & 2.428519 \\
\hline 35 & 1 & 0 & 5.625559 & -0.585055 & -0.510830 \\
\hline 36 & 1 & 0 & 5.886254 & 0.778638 & -1.618199 \\
\hline 37 & 1 & 0 & 4.174704 & 0.859935 & 2.588669 \\
\hline
\end{tabular}




$\begin{array}{lllrrl}38 & 1 & 0 & 5.540804 & -0.849521 & -2.276250 \\ 39 & 1 & 0 & 1.607168 & 1.163232 & -1.783151 \\ 40 & 1 & 0 & -2.040777 & -2.439744 & -0.644929 \\ 41 & 1 & 0 & -5.363575 & 1.166321 & -0.035871 \\ 42 & 1 & 0 & -4.445265 & -2.999574 & -0.605456 \\ 43 & 1 & 0 & -6.142762 & -1.191059 & -0.303213\end{array}$

$\mathrm{W}=-404.0872$

$\mathrm{HF}=-1090.8481854$

Sum of electronic and zero-point Energies= $\quad-1090.504390$

Sum of electronic and thermal Energies= $\quad-1090.483618$

Sum of electronic and thermal Enthalpies $=\quad-1090.482673$

Sum of electronic and thermal Free Energies $=\quad-1090.554306$

Table S6 Cartesian coordinates, energy and imaginary frequency of B3LYP/6-31G* optimization of transition state from compound $5 \mathrm{E}$ to 6out.

Standard orientation:

\begin{tabular}{|c|c|c|c|c|}
\hline Center & Atomic & Atomic & Coordinate & s (Angstrc \\
\hline Number & Numbe & Type & $X$ & $\mathrm{Z}$ \\
\hline 1 & 6 & 4.074967 & -2.215187 & -0.227765 \\
\hline 2 & 6 & 5.067125 & -1.291056 & -0.560387 \\
\hline 3 & 6 & 4.782107 & 0.066134 & -0.485869 \\
\hline 4 & 6 & 3.504595 & 0.489435 & -0.106138 \\
\hline 5 & 6 & 2.480043 & -0.413880 & 0.243488 \\
\hline 6 & 6 & 2.816168 & -1.776268 & 0.175238 \\
\hline 7 & 6 & 1.078571 & -0.026688 & 0.697952 \\
\hline 8 & 6 & 0.050435 & 0.062708 & -0.515642 \\
\hline 9 & 6 & -1.305775 & 0.224914 & 0.153526 \\
\hline 10 & 6 & -1.613381 & -1.209772 & 0.576744 \\
\hline 11 & 6 & -0.686464 & -1.464673 & 1.772887 \\
\hline 12 & 6 & 0.549485 & -0.949845 & 1.787516 \\
\hline 13 & 6 & -0.095722 & -1.280858 & -1.207383 \\
\hline 14 & 6 & -1.043347 & -2.002131 & -0.600540 \\
\hline 15 & 6 & -1.764368 & 1.404186 & 0.708814 \\
\hline 16 & 6 & -3.017661 & 1.516797 & 1.320666 \\
\hline 17 & 8 & -4.251836 & 1.510985 & -0.170751 \\
\hline 18 & 6 & -3.993350 & 0.488419 & -0.895860 \\
\hline 19 & 8 & -4.663989 & -0.698271 & -0.650568 \\
\hline 20 & 6 & -5.893708 & -0.575620 & 0.061069 \\
\hline
\end{tabular}




$\begin{array}{rrrrrr}21 & 7 & 0 & 3.308232 & 1.950393 & -0.084509 \\ 22 & 8 & 0 & 2.171192 & 2.396914 & -0.259478 \\ 23 & 6 & 0 & -2.947097 & 0.418395 & -1.790090 \\ 24 & 8 & 0 & 4.301575 & 2.654760 & 0.091182 \\ 25 & 1 & 0 & -3.338073 & 2.476321 & 1.709730 \\ 26 & 1 & 0 & -2.659105 & -1.423583 & 0.800127 \\ 27 & 1 & 0 & -1.333370 & -3.023502 & -0.825447 \\ 28 & 1 & 0 & 0.346849 & 0.893303 & -1.158407 \\ 29 & 1 & 0 & 0.533481 & -1.603820 & -2.029881 \\ 30 & 1 & 0 & 1.114306 & 0.985257 & 1.112226 \\ 31 & 1 & 0 & 1.221538 & -1.154698 & 2.619301 \\ 32 & 1 & 0 & -1.043098 & -2.087434 & 2.590711 \\ 33 & 1 & 0 & -2.802611 & -0.462423 & -2.402364 \\ 34 & 1 & 0 & -1.260660 & 2.322149 & 0.408348 \\ 35 & 1 & 0 & -6.579911 & 0.111525 & -0.447330 \\ 36 & 1 & 0 & -5.745032 & -0.218860 & 1.085454 \\ 37 & 1 & 0 & -3.459872 & 0.657014 & 1.812658 \\ 38 & 1 & 0 & -6.324822 & -1.579140 & 0.080914 \\ 39 & 1 & 0 & -2.458808 & 1.337373 & -2.081878 \\ 40 & 1 & 0 & 2.055459 & -2.499670 & 0.450688 \\ 41 & 1 & 0 & 5.529098 & 0.814657 & -0.719342 \\ 42 & 1 & 0 & 4.282112 & -3.280660 & -0.275920 \\ 43 & 1 & 0 & 6.053614 & -1.621735 & -0.870470 \\ & 1 & & & & \end{array}$

$\mathrm{W}=-407.2356$

$\mathrm{HF}=-1090.8539303$

Sum of electronic and zero-point Energies $=\quad-1090.510159$

Sum of electronic and thermal Energies= $\quad-1090.489347$

Sum of electronic and thermal Enthalpies $=\quad-1090.488403$

Sum of electronic and thermal Free Energies $=\quad-1090.560276$

Table S7 Cartesian coordinates, energy and imaginary frequency of B3LYP/6-31G* optimization of transition state from compound $5 \mathrm{Z}$ to $6 \mathrm{in}$.

Standard orientation:

\begin{tabular}{|c|c|c|c|c|}
\hline Center & Atomic & Atomic & Coordinates & s (Angstr \\
\hline Number & Numbe & Type & $\mathrm{X}$ & $\mathrm{Z}$ \\
\hline 1 & 6 & 0 & 744 & 994 \\
\hline 2 & 6 & 4.749145 & -0.056942 & -0.165946 \\
\hline 3 & 6 & 3.921747 & 1.055425 & -0.262689 \\
\hline
\end{tabular}




\begin{tabular}{cccccc}
4 & 6 & 0 & 2.542777 & 0.906500 & -0.097589 \\
5 & 6 & 0 & 1.937800 & -0.325128 & 0.216602 \\
6 & 6 & 0 & 2.811440 & -1.419876 & 0.322100 \\
7 & 6 & 0 & 0.448495 & -0.520189 & 0.504715 \\
8 & 6 & 0 & -0.380401 & -0.883393 & -0.780892 \\
9 & 6 & 0 & -1.773687 & -1.386511 & -0.393789 \\
10 & 6 & 0 & -1.449791 & -2.749974 & 0.211232 \\
11 & 6 & 0 & -0.644498 & -2.560297 & 1.492706 \\
12 & 6 & 0 & 0.235823 & -1.559082 & 1.596913 \\
13 & 6 & 0 & 0.131485 & -2.175912 & -1.420713 \\
14 & 6 & 0 & -0.473155 & -3.236046 & -0.871863 \\
15 & 6 & 0 & -2.946274 & -1.108135 & -1.074853 \\
16 & 6 & 0 & -3.212057 & 0.131082 & -1.659674 \\
17 & 8 & 0 & -3.711800 & 1.245619 & -0.121735 \\
18 & 6 & 0 & -2.818523 & 1.138126 & 0.784925 \\
19 & 8 & 0 & -1.827882 & 2.090224 & 0.890880 \\
20 & 6 & 0 & -2.031814 & 3.305064 & 0.169780 \\
21 & 7 & 0 & 1.753310 & 2.133968 & -0.289920 \\
22 & 8 & 0 & 2.239247 & 3.193751 & 0.102759 \\
23 & 6 & 0 & -2.669074 & 0.026723 & 1.593695 \\
24 & 8 & 0 & 0.668418 & 2.038582 & -0.866185 \\
25 & 1 & 0 & -4.152952 & 0.291316 & -2.172418 \\
26 & 1 & 0 & -2.323911 & -3.390363 & 0.358249 \\
27 & 1 & 0 & -0.281342 & -4.283381 & -1.084833 \\
28 & 1 & 0 & -0.378356 & -0.029020 & -1.455533 \\
29 & 1 & 0 & 0.910052 & -2.197683 & -2.176552 \\
30 & 1 & 0 & 0.036184 & 0.430933 & 0.859233 \\
31 & 1 & 0 & 0.827757 & -1.440267 & 2.503134 \\
32 & 1 & 0 & -0.783881 & -3.269502 & 2.305505 \\
33 & 1 & 0 & -1.932103 & 0.018612 & 2.385900 \\
34 & 1 & 0 & -3.791070 & -1.775213 & -0.905557 \\
35 & 1 & 0 & -2.919139 & 3.832947 & 0.538932 \\
36 & 1 & 0 & -2.147235 & 3.129079 & -0.902799 \\
37 & 1 & 0 & -2.399106 & 0.773560 & -1.979421 \\
38 & 1 & 0 & -1.135167 & 3.901192 & 0.342570 \\
39 & 1 & 0 & -3.481865 & -0.683217 & 1.647558 \\
40 & 1 & 0 & 2.387715 & -2.385555 & 0.578726 \\
41 & 1 & 0 & 4.319385 & 2.041317 & -0.472699 \\
42 & 1 & 0 & 4.817704 & -2.180674 & 0.203922 \\
43 & 1 & 0 & 5.819962 & 0.048594 & -0.310744 \\
-------------------------------------------------------- \\
\hline
\end{tabular}

$\mathrm{W}=-394.7706$

$\mathrm{HF}=-1090.8487515$ 
Sum of electronic and zero-point Energies= $\quad-1090.504912$

Sum of electronic and thermal Energies= $\quad-1090.484109$

Sum of electronic and thermal Enthalpies= $\quad-1090.483165$

Sum of electronic and thermal Free Energies $=\quad-1090.554551$

Table S8 Cartesian coordinates, energy and imaginary frequency of B3LYP/6-31G* optimization of transition state from compound $5 \mathrm{Z}$ to 6out.

Standard orientation:

\begin{tabular}{|c|c|c|c|c|c|}
\hline \multirow{2}{*}{$\begin{array}{l}\text { Center } \\
\text { Number }\end{array}$} & \multirow{2}{*}{$\begin{array}{l}\text { Atomic } \\
\text { Number }\end{array}$} & \multirow{2}{*}{\multicolumn{2}{|c|}{$\begin{array}{l}\text { Atomic } \\
\text { Type }\end{array}$}} & \multicolumn{2}{|c|}{ Coordinates (Angstrom } \\
\hline & & & & $\mathrm{X}$ & $\mathrm{Z}$ \\
\hline 1 & 6 & 0 & 3.953110 & 1.148245 & -0.440958 \\
\hline 2 & 6 & 0 & 4.628690 & 0.127185 & -1.096262 \\
\hline 3 & 6 & 0 & 4.059072 & -1.146554 & -1.143263 \\
\hline 4 & 6 & 0 & 2.829177 & -1.386296 & -0.535470 \\
\hline 5 & 6 & 0 & 2.104288 & -0.378043 & 0.121533 \\
\hline 6 & 6 & 0 & 2.706260 & 0.897191 & 0.140794 \\
\hline 7 & 6 & 0 & 0.770090 & -0.733364 & 0.763765 \\
\hline 8 & 6 & 0 & -0.450973 & -0.554375 & -0.238599 \\
\hline 9 & 6 & 0 & -1.636133 & -1.164231 & 0.501504 \\
\hline 10 & 6 & 0 & -1.403742 & -2.653570 & 0.275103 \\
\hline 11 & 6 & 0 & -0.227453 & -3.003457 & 1.198680 \\
\hline 12 & 6 & 0 & 0.772178 & -2.129673 & 1.372256 \\
\hline 13 & 6 & 0 & -0.318434 & -1.486086 & -1.432321 \\
\hline 14 & 6 & 0 & -0.866525 & -2.672981 & -1.154833 \\
\hline 15 & 6 & 0 & -2.339640 & -0.625818 & 1.561400 \\
\hline 16 & 6 & 0 & -2.506563 & 0.756189 & 1.723992 \\
\hline 17 & 8 & 0 & -3.796277 & 1.218534 & 0.374230 \\
\hline 18 & 6 & 0 & -3.382781 & 0.773834 & -0.754178 \\
\hline 19 & 8 & 0 & -2.629783 & 1.594337 & -1.570553 \\
\hline 20 & 6 & 0 & -2.645620 & 2.984632 & -1.247504 \\
\hline 21 & 7 & 0 & 2.071068 & 2.059983 & 0.786501 \\
\hline 22 & 8 & 0 & 0.839999 & 2.087690 & 0.878245 \\
\hline 23 & 6 & 0 & -3.481316 & -0.544907 & -1.145774 \\
\hline 24 & 8 & 0 & 2.806313 & 2.960186 & 1.188895 \\
\hline 25 & 1 & 0 & -1.741053 & 1.439732 & 1.374255 \\
\hline 26 & 1 & 0 & -2.272507 & -3.290490 & 0.461659 \\
\hline 27 & 1 & 0 & -0.868961 & -3.556976 & -1.784686 \\
\hline 28 & 1 & 0 & -0.550405 & 0.497958 & -0.499679 \\
\hline 29 & 1 & 0 & 0.201988 & -1.214438 & -2.344467 \\
\hline 30 & 1 & 0 & 0.576686 & -0.026134 & 1.575806 \\
\hline 31 & 1 & 0 & 1.622798 & -2.386176 & 2.001691 \\
\hline 32 & 1 & 0 & -0.213387 & -3.976375 & 1.685329 \\
\hline
\end{tabular}




$\begin{array}{rrrrrr}33 & 1 & 0 & -4.154015 & -1.192434 & -0.601374 \\ 34 & 1 & 0 & -3.015118 & -1.286858 & 2.103066 \\ 35 & 1 & 0 & -2.096866 & 3.197350 & -0.324274 \\ 36 & 1 & 0 & -3.669994 & 3.356913 & -1.140783 \\ 37 & 1 & 0 & -3.096887 & 1.124729 & 2.555159 \\ 38 & 1 & 0 & -2.152917 & 3.485149 & -2.084102 \\ 39 & 1 & 0 & -3.143924 & -0.854790 & -2.126603 \\ 40 & 1 & 0 & 2.400509 & -2.383120 & -0.560732 \\ 41 & 1 & 0 & 4.575153 & -1.957721 & -1.649374 \\ 42 & 1 & 0 & 4.367800 & 2.146125 & -0.370440 \\ 43 & 1 & 0 & 5.589509 & 0.324186 & -1.561697 \\ -\end{array}$

$\mathrm{W}=-412.4956$

$\mathrm{HF}=-1090.8548027$

Sum of electronic and zero-point Energies= $\quad-1090.510949$

Sum of electronic and thermal Energies $=\quad-1090.490153$

Sum of electronic and thermal Enthalpies $=\quad-1090.489208$

Sum of electronic and thermal Free Energies $=\quad-1090.561126$

Table S9 Cartesian coordinates, energy and imaginary frequency of B3LYP/6-31G* optimization of transition state from compound 7E to 8in.

Standard orientation:

\begin{tabular}{|c|c|c|c|c|}
\hline \multirow{2}{*}{$\begin{array}{l}\text { Center } \\
\text { Number }\end{array}$} & \multirow{2}{*}{$\begin{array}{l}\text { Atomic } \\
\text { Number }\end{array}$} & \multirow{2}{*}{$\begin{array}{r}\text { Atomic } \\
\text { Type }\end{array}$} & \multicolumn{2}{|c|}{ Coordinates (Angstroms) } \\
\hline & & & $\mathrm{X}$ & $\mathrm{Z}$ \\
\hline 1 & 6 & -1.303079 & 0.720634 & 0.734187 \\
\hline 2 & 6 & 0.955633 & 0.403319 & -0.351478 \\
\hline 3 & 6 & 0.355973 & 0.288571 & -1.544106 \\
\hline 4 & 6 & -3.482576 & 0.485907 & 1.826818 \\
\hline 5 & 1 & -4.052479 & 0.875831 & 0.990009 \\
\hline 6 & 1 & -4.038900 & 0.411086 & 2.754142 \\
\hline 7 & 6 & -2.093566 & 0.662634 & 1.873416 \\
\hline 8 & 1 & -1.594041 & 0.439444 & 2.815683 \\
\hline 9 & 6 & -1.606350 & -1.752953 & 0.316919 \\
\hline 10 & 1 & -1.135818 & -1.944617 & 1.270992 \\
\hline 11 & 1 & -0.169926 & 4.573911 & -1.293936 \\
\hline 12 & 8 & -3.681077 & -1.352671 & 1.341427 \\
\hline 13 & 6 & -2.976358 & -1.585833 & 0.297247 \\
\hline 14 & 8 & -3.563145 & -1.481554 & -0.948786 \\
\hline 15 & 1 & 0.044517 & 3.039213 & 1.707187 \\
\hline
\end{tabular}




$\begin{array}{rrrrrr}16 & 1 & 0 & -1.060450 & -2.022676 & -0.577817 \\ 17 & 1 & 0 & 0.896891 & -0.146284 & -2.378894 \\ 18 & 6 & 0 & -1.071661 & 0.699980 & -1.788344 \\ 19 & 8 & 0 & 1.012670 & 3.252531 & -0.151713 \\ 20 & 1 & 0 & 0.213690 & 3.024167 & -2.097782 \\ 21 & 1 & 0 & -1.129839 & 1.351460 & -2.671850 \\ 22 & 6 & 0 & 2.383374 & 0.038818 & -0.102944 \\ 23 & 1 & 0 & -1.870372 & 3.494766 & 0.102614 \\ 24 & 1 & 0 & -2.783434 & 1.463333 & -0.716658 \\ 25 & 1 & 0 & 0.625119 & 0.692351 & 1.779784 \\ 26 & 1 & 0 & -1.672162 & -0.184972 & -2.034264 \\ 27 & 6 & 0 & 0.083573 & 2.570768 & 0.717507 \\ 28 & 6 & 0 & -4.988527 & -1.527921 & -0.973956 \\ 29 & 1 & 0 & -5.437802 & -0.646654 & -0.503898 \\ 30 & 1 & 0 & -5.365732 & -2.421988 & -0.465538 \\ 31 & 1 & 0 & -5.263491 & -1.558898 & -2.030425 \\ 32 & 6 & 0 & -1.702140 & 1.407319 & -0.574942 \\ 33 & 6 & 0 & -1.097733 & 2.814139 & -0.261272 \\ 34 & 6 & 0 & -0.022324 & 3.498305 & -1.140011 \\ 35 & 6 & 0 & 0.190976 & 1.011232 & 0.828762 \\ 36 & 6 & 0 & 5.085714 & -0.510134 & 0.596267 \\ 37 & 6 & 0 & 2.892525 & -1.272916 & -0.044074 \\ 38 & 6 & 0 & 3.290830 & 1.067772 & 0.212887 \\ 39 & 6 & 0 & 4.619258 & 0.804893 & 0.538263 \\ 40 & 6 & 0 & 4.211191 & -1.553977 & 0.320459 \\ 41 & 7 & 0 & 2.059916 & -2.450154 & -0.336908 \\ 42 & 1 & 0 & 2.926825 & 2.089635 & 0.173849 \\ 43 & 1 & 0 & 5.291322 & 1.631594 & 0.751582 \\ 44 & 1 & 0 & 4.529941 & -2.588677 & 0.375316 \\ 45 & 1 & 0 & 6.117891 & -0.722814 & 0.857893 \\ 46 & 8 & 0 & 2.137047 & -3.398945 & 0.444309 \\ 47 & 8 & 0 & 1.364562 & -2.434546 & -1.352646 \\ ----------------------------------------------------------- \\ & & & & \end{array}$

$W=-426.0299$

$\mathrm{HF}=-1205.3764202$

Sum of electronic and zero-point Energies $=\quad-1204.996879$

Sum of electronic and thermal Energies= $\quad-1204.974624$

Sum of electronic and thermal Enthalpies= $\quad-1204.973680$

Sum of electronic and thermal Free Energies $=\quad-1205.048652$ 
Table S10 Cartesian coordinates, energy and imaginary frequency of B3LYP/6-31+G** optimization of transition state from compound 7E to 8in.

Standard orientation:

\begin{tabular}{lrrrrc} 
Center & \multicolumn{2}{c}{ Atomic } & \multicolumn{2}{c}{ Atomic } & \multicolumn{3}{c}{ Coordinates } \\
Number & Number & Type & X & Y & Z \\
---------------- \\
1 & 6 & 0 & -1.292022 & 0.747464 & 0.745668 \\
2 & 6 & 0 & 0.961788 & 0.401337 & -0.338405 \\
3 & 6 & 0 & 0.356727 & 0.275277 & -1.528553 \\
4 & 6 & 0 & -3.473968 & 0.517568 & 1.846382 \\
5 & 1 & 0 & -4.043840 & 0.905732 & 1.009657 \\
6 & 1 & 0 & -4.025137 & 0.424093 & 2.774078 \\
7 & 6 & 0 & -2.081359 & 0.662138 & 1.883597 \\
8 & 1 & 0 & -1.579139 & 0.426750 & 2.820779 \\
9 & 6 & 0 & -1.626473 & -1.784208 & 0.324734 \\
10 & 1 & 0 & -1.159890 & -1.971813 & 1.281039 \\
11 & 1 & 0 & -0.153050 & 4.577907 & -1.318895 \\
12 & 8 & 0 & -3.702770 & -1.349817 & 1.338188 \\
13 & 6 & 0 & -2.992317 & -1.585646 & 0.298078 \\
14 & 8 & 0 & -3.568129 & -1.456333 & -0.952527 \\
15 & 1 & 0 & 0.076775 & 3.068657 & 1.692277 \\
16 & 1 & 0 & -1.081971 & -2.045995 & -0.572690 \\
17 & 1 & 0 & 0.892547 & -0.172108 & -2.360007 \\
18 & 6 & 0 & -1.067808 & 0.696773 & -1.773815 \\
19 & 8 & 0 & 1.032041 & 3.258140 & -0.176519 \\
20 & 1 & 0 & 0.217688 & 3.019026 & -2.115244 \\
21 & 1 & 0 & -1.120075 & 1.339414 & -2.663199 \\
22 & 6 & 0 & 2.387570 & 0.030408 & -0.089484 \\
23 & 1 & 0 & -1.851599 & 3.514660 & 0.094057 \\
24 & 1 & 0 & -2.774215 & 1.481511 & -0.705391 \\
25 & 1 & 0 & 0.635105 & 0.717544 & 1.790071 \\
26 & 1 & 0 & -1.674672 & -0.185309 & -2.011788 \\
27 & 6 & 0 & 0.103864 & 2.589380 & 0.708274 \\
28 & 6 & 0 & -4.995688 & -1.520467 & -1.008028 \\
29 & 1 & 0 & -5.461691 & -0.691835 & -0.466792 \\
30 & 1 & 0 & -5.361292 & -2.464139 & -0.589421 \\
31 & 1 & 0 & -5.249765 & -1.460625 & -2.067641 \\
32 & 6 & 0 & -1.693340 & 1.421929 & -0.566548 \\
33 & 6 & 0 & -1.082235 & 2.829807 & -0.267580 \\
34 & 6 & 0 & -0.011966 & 3.503082 & -1.161504 \\
35 & 6 & 0 & 0.203109 & 1.028624 & 0.835988 \\
36 & 6 & 0 & 5.093243 & -0.546930 & 0.585227
\end{tabular}




$\begin{array}{rllrrc}37 & 6 & 0 & 2.888635 & -1.284266 & -0.042423 \\ 38 & 6 & 0 & 3.306273 & 1.050062 & 0.229284 \\ 39 & 6 & 0 & 4.635747 & 0.773365 & 0.543562 \\ 40 & 6 & 0 & 4.207775 & -1.583044 & 0.307215 \\ 41 & 7 & 0 & 2.036094 & -2.449540 & -0.336853 \\ 42 & 1 & 0 & 2.952804 & 2.075567 & 0.199576 \\ 43 & 1 & 0 & 5.315286 & 1.592740 & 0.758590 \\ 44 & 1 & 0 & 4.520767 & -2.620261 & 0.350664 \\ 45 & 1 & 0 & 6.125537 & -0.769315 & 0.835831 \\ 46 & 8 & 0 & 2.007408 & -3.351763 & 0.502811 \\ 47 & 8 & 0 & 1.429179 & -2.469145 & -1.407902 \\ -\end{array}$

$\mathrm{W}=-396.8855$

$\mathrm{HF}=-1205.4499345$

Sum of electronic and zero-point Energies $=\quad-1205.072698$

Sum of electronic and thermal Energies $=\quad-1205.050209$

Sum of electronic and thermal Enthalpies $=\quad-1205.049265$

Sum of electronic and thermal Free Energies $=\quad-1205.125005$

Table S11 Cartesian coordinates and energy of MPW1K/6-31+G** optimization of transition state from compound $7 \mathrm{E}$ to 8in.

Standard orientation:

\begin{tabular}{|c|c|c|c|c|}
\hline \multirow{2}{*}{$\begin{array}{l}\text { Center } \\
\text { Number }\end{array}$} & \multirow{2}{*}{$\begin{array}{l}\text { Atomic } \\
\text { Number }\end{array}$} & \multirow{2}{*}{$\begin{array}{r}\text { Atomic } \\
\text { Type }\end{array}$} & \multicolumn{2}{|c|}{ Coordinates (Angstroms) } \\
\hline & & & $\mathrm{X}$ & $\mathrm{Z}$ \\
\hline 1 & 6 & 1.315758 & 0.664272 & -0.704163 \\
\hline 2 & 6 & -0.939334 & 0.394082 & 0.357188 \\
\hline 3 & 6 & -0.361775 & 0.273759 & 1.549238 \\
\hline 4 & 6 & 3.479587 & 0.442810 & -1.766879 \\
\hline 5 & 1 & 4.037246 & 0.834882 & -0.928049 \\
\hline 6 & 1 & 4.046179 & 0.402857 & -2.683669 \\
\hline 7 & 6 & 2.102651 & 0.686078 & -1.842071 \\
\hline 8 & 1 & 1.613721 & 0.498638 & -2.789625 \\
\hline 9 & 6 & 1.542529 & -1.601583 & -0.328475 \\
\hline 10 & 1 & 1.079654 & -1.806745 & -1.276841 \\
\hline 11 & 1 & 0.191081 & 4.475446 & 1.311346 \\
\hline 12 & 8 & 3.611828 & -1.281829 & -1.341742 \\
\hline 13 & 6 & 2.911554 & -1.517826 & -0.302041 \\
\hline 14 & 8 & 3.511008 & -1.450078 & 0.910771 \\
\hline 15 & 1 & 0.032053 & 2.972503 & -1.688342 \\
\hline
\end{tabular}




\begin{tabular}{|c|c|c|c|c|c|}
\hline 16 & 1 & 0 & 0.998532 & -1.867792 & 0.561979 \\
\hline 17 & 1 & 0 & -0.921395 & -0.144042 & 2.373217 \\
\hline 18 & 6 & 0 & 1.059606 & 0.655052 & 1.801617 \\
\hline 19 & 8 & 0 & -0.958473 & 3.192180 & 0.136559 \\
\hline 20 & 1 & 0 & -0.223614 & 2.924312 & 2.075607 \\
\hline 21 & 1 & 0 & 1.119620 & 1.306704 & 2.675909 \\
\hline 22 & 6 & 0 & -2.360430 & 0.052749 & 0.097772 \\
\hline 23 & 1 & 0 & 1.896988 & 3.410107 & -0.059599 \\
\hline 24 & 1 & 0 & 2.777536 & 1.384998 & 0.752343 \\
\hline 25 & 1 & 0 & -0.591514 & 0.651647 & -1.756812 \\
\hline 26 & 1 & 0 & 1.634918 & -0.234943 & 2.061208 \\
\hline 27 & 6 & 0 & -0.035045 & 2.510637 & -0.705164 \\
\hline 28 & 6 & 0 & 4.912537 & -1.605892 & 0.927803 \\
\hline 29 & 1 & 0 & 5.417826 & -0.784506 & 0.425768 \\
\hline 30 & 1 & 0 & 5.205019 & -2.539069 & 0.450434 \\
\hline 31 & 1 & 0 & 5.196017 & -1.624146 & 1.974382 \\
\hline 32 & 6 & 0 & 1.702802 & 1.339445 & 0.602989 \\
\hline 33 & 6 & 0 & 1.120435 & 2.734957 & 0.285409 \\
\hline 34 & 6 & 0 & 0.039042 & 3.411065 & 1.138598 \\
\hline 35 & 6 & 0 & -0.163476 & 0.971624 & -0.810071 \\
\hline 36 & 6 & 0 & -5.043448 & -0.468095 & -0.611577 \\
\hline 37 & 6 & 0 & -2.877783 & -1.240861 & 0.033878 \\
\hline 38 & 6 & 0 & -3.247224 & 1.083388 & -0.222988 \\
\hline 39 & 6 & 0 & -4.567188 & 0.833580 & -0.553396 \\
\hline 40 & 6 & 0 & -4.186186 & -1.513294 & -0.332668 \\
\hline 41 & 7 & 0 & -2.060015 & -2.408828 & 0.328966 \\
\hline 42 & 1 & 0 & -2.872345 & 2.095435 & -0.185594 \\
\hline 43 & 1 & 0 & -5.225705 & 1.661086 & -0.771703 \\
\hline 44 & 1 & 0 & -4.511759 & -2.539903 & -0.389474 \\
\hline 45 & 1 & 0 & -6.069939 & -0.669136 & -0.876886 \\
\hline 46 & 8 & 0 & -2.087333 & -3.314942 & -0.473155 \\
\hline 47 & 8 & 0 & -1.431741 & -2.418108 & 1.361522 \\
\hline
\end{tabular}

$\mathrm{HF}=-1205.0782317$

Table S12 Energetics of IEFPCM/B3LYP/6-31+G**//B3LYP/6-31+G** $\varepsilon=$ toluene single point calculation of transition state from compound $7 \mathrm{E}$ to 8 in.

Variational PCM results

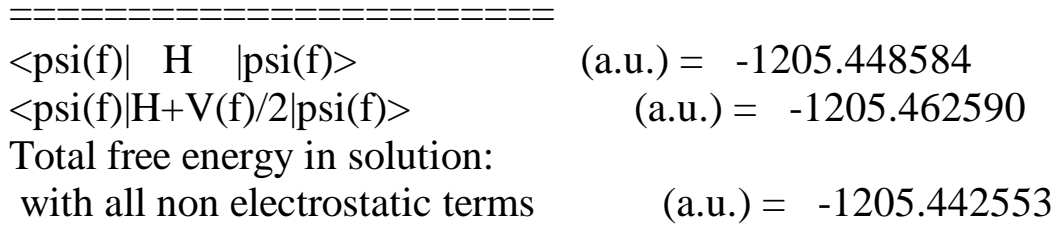




\begin{tabular}{lcc} 
(Polarized solute)-Solvent & $(\mathrm{kcal} / \mathrm{mol})=$ & -8.79 \\
\hline-------- & \\
Cavitation energy & $(\mathrm{kcal} / \mathrm{mol})=$ & 42.48 \\
Dispersion energy & $(\mathrm{kcal} / \mathrm{mol})=$ & -36.23 \\
Repulsion energy & $(\mathrm{kcal} / \mathrm{mol})=$ & 6.32 \\
Total non electrostatic & $(\mathrm{kcal} / \mathrm{mol})=$ & 12.57
\end{tabular}

Table S13 Energetics of IEFPCM/B3LYP/6-31+G**//B3LYP/6-31+G** $\varepsilon=$ water single point calculation of transition state from compound $7 \mathrm{E}$ to $8 \mathrm{in}$.

Variational PCM results

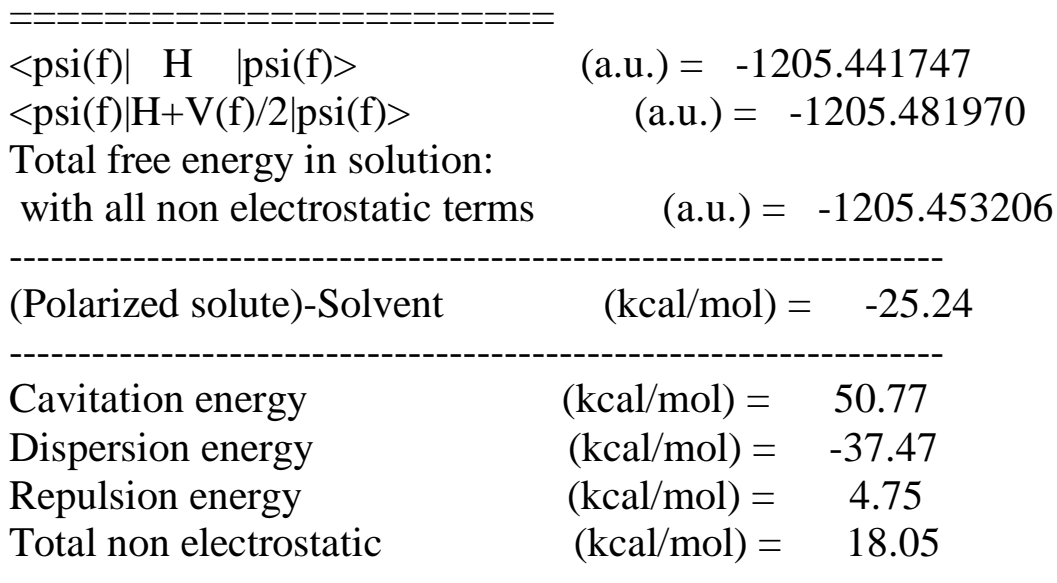

Table S14 Energetics of IEFPCM/ MPW1K/6-31+G**//MPW1K/6-31+G** $\varepsilon=$ toluene single point calculation of transition state from compound $7 \mathrm{E}$ to $8 \mathrm{in}$.

Variational PCM results

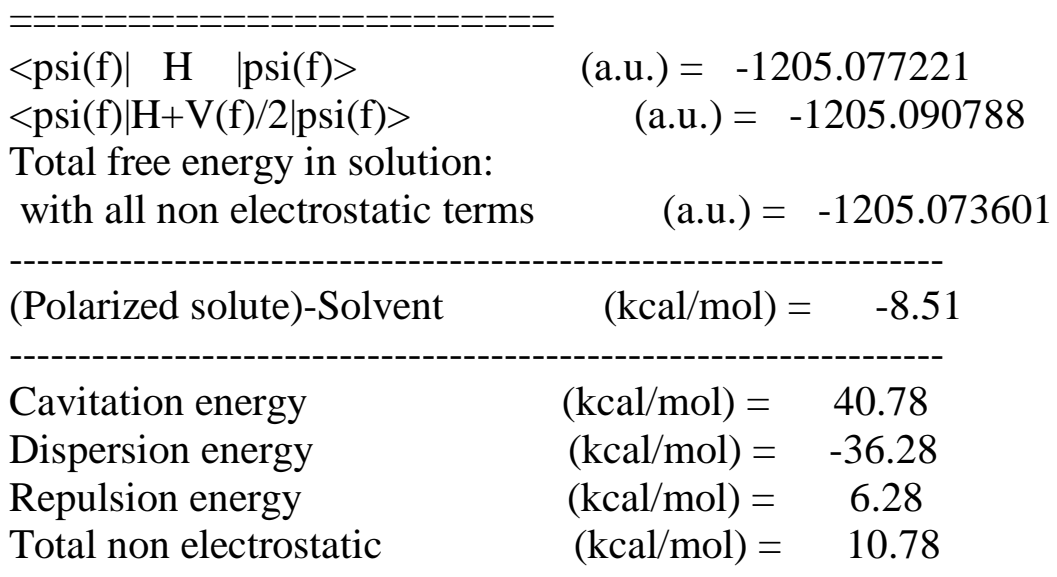


Table S15 Energetics of IEFPCM/ MPW1K/6-31+G**//MPW1K/6-31+G** $\varepsilon=$ water single point calculation of transition state from compound $7 \mathrm{E}$ to $8 \mathrm{in}$.

Variational PCM results

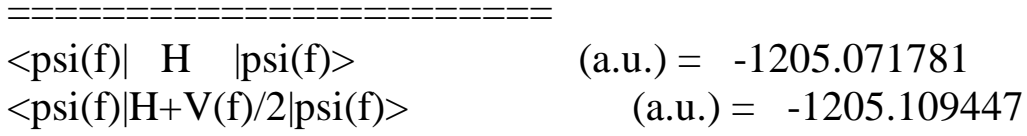

Total free energy in solution:

with all non electrostatic terms $\quad$ (a.u.) $=-1205.084086$

(Polarized solute)-Solvent $\quad(\mathrm{kcal} / \mathrm{mol})=-23.64$

Cavitation energy

Dispersion energy

Repulsion energy

Total non electrostatic $(\mathrm{kcal} / \mathrm{mol})=48.71$

$(\mathrm{kcal} / \mathrm{mol})=-37.50$

$(\mathrm{kcal} / \mathrm{mol})=4.71$

$(\mathrm{kcal} / \mathrm{mol})=15.91$

Table S16 Cartesian coordinates, energy and imaginary frequency of B3LYP/6-31G* optimization of transition state from compound $7 \mathrm{E}$ to 8out.

Standard orientation:

\begin{tabular}{cccccc} 
Center & \multicolumn{2}{c}{ Atomic } & \multicolumn{2}{c}{ Atomic } & \multicolumn{3}{c}{ Coordinates } \\
Number & Number & Type & X & Y & Z \\
- & Nums & \\
1 & 6 & 0 & 1.597112 & 0.667463 & 1.018380 \\
2 & 6 & 0 & 1.139864 & -0.359695 & -0.009378 \\
3 & 1 & 0 & 2.737608 & -3.320550 & 0.519766 \\
4 & 6 & 0 & -0.077651 & 0.299956 & -0.637384 \\
5 & 6 & 0 & 0.600027 & 0.535012 & 2.202298 \\
6 & 6 & 0 & 1.490675 & 1.994776 & 0.220430 \\
7 & 1 & 0 & 2.615697 & 0.510922 & 1.377424 \\
8 & 6 & 0 & 0.549308 & 3.151693 & 0.625932 \\
9 & 1 & 0 & 0.062510 & 3.085486 & 1.604994 \\
10 & 1 & 0 & 2.479857 & 2.317373 & -0.106730 \\
11 & 1 & 0 & -0.438225 & -0.206774 & -1.534033 \\
12 & 6 & 0 & 0.451321 & 1.742591 & -0.900468 \\
13 & 6 & 0 & -1.173474 & 0.316367 & 0.442845 \\
14 & 1 & 0 & 0.755329 & 1.883284 & -1.941859 \\
15 & 8 & 0 & -0.372477 & 2.849216 & -0.451937 \\
16 & 1 & 0 & 0.979282 & 4.153436 & 0.505124 \\
17 & 6 & 0 & -2.607539 & 0.313228 & 0.014620 \\
18 & 1 & 0 & 2.407568 & -0.664822 & -2.515369
\end{tabular}




\begin{tabular}{rrrrrr}
19 & 1 & 0 & -1.608493 & 0.478218 & 2.496189 \\
20 & 6 & 0 & 2.555627 & -2.253798 & 0.573384 \\
21 & 6 & 0 & -0.832949 & 0.444962 & 1.732806 \\
22 & 1 & 0 & 3.019146 & 1.048640 & -2.132583 \\
23 & 1 & 0 & 0.846252 & -2.342351 & -0.699655 \\
24 & 1 & 0 & 6.448898 & -0.738985 & -0.297240 \\
25 & 1 & 0 & 5.484730 & -0.882930 & 1.187488 \\
26 & 8 & 0 & 3.983087 & -1.796148 & -0.744377 \\
27 & 6 & 0 & 2.966105 & -0.001153 & -1.870328 \\
28 & 1 & 0 & 3.052121 & -1.743986 & 1.390753 \\
29 & 1 & 0 & 6.318160 & 0.655121 & 0.817886 \\
30 & 6 & 0 & 5.787688 & -0.197987 & 0.389106 \\
31 & 6 & 0 & 3.899712 & -0.554461 & -1.015037 \\
32 & 8 & 0 & 4.666557 & 0.351995 & -0.300793 \\
33 & 6 & 0 & 1.413149 & -1.714879 & -0.017870 \\
34 & 1 & 0 & 0.853217 & -0.367694 & 2.777416 \\
35 & 1 & 0 & 0.720568 & 1.376955 & 2.898248 \\
36 & 6 & 0 & -5.357930 & 0.452614 & -0.671960 \\
37 & 6 & 0 & -3.409291 & -0.836978 & -0.098649 \\
38 & 6 & 0 & -3.236630 & 1.541879 & -0.244471 \\
39 & 6 & 0 & -4.586172 & 1.613164 & -0.588847 \\
40 & 6 & 0 & -4.767314 & -0.779837 & -0.417534 \\
41 & 7 & 0 & -2.845086 & -2.178740 & 0.105414 \\
42 & 1 & 0 & -2.630816 & 2.439236 & -0.180794 \\
43 & 1 & 0 & -5.036746 & 2.581970 & -0.786395 \\
44 & 1 & 0 & -5.332044 & -1.703049 & -0.465140 \\
45 & 1 & 0 & -6.410211 & 0.504096 & -0.934825 \\
46 & 8 & 0 & -3.603579 & -3.067300 & 0.492917 \\
47 & 8 & 0 & -1.650920 & -2.345501 & -0.146535 \\
--------------------------------------------------------------- \\
\hline
\end{tabular}

$\mathrm{W}=-358.5710$

$\mathrm{HF}=-1205.3834372$

Sum of electronic and zero-point Energies $=\quad-1205.004417$

Sum of electronic and thermal Energies= $\quad-1204.982016$

Sum of electronic and thermal Enthalpies $=\quad-1204.981072$

Sum of electronic and thermal Free Energies $=\quad-1205.056904$

Table S17 Cartesian coordinates and energy of B3LYP/6-31+G** optimization of transition state from compound 7E to 8out.

Standard orientation:

Center Atomic Atomic Coordinates (Angstroms)




\begin{tabular}{|c|c|c|c|c|c|}
\hline Number & & Number & Type & $\mathrm{X}$ & $\mathrm{Z}$ \\
\hline 1 & 6 & 0 & 1.570712 & 0.582570 & 1.073628 \\
\hline 2 & 6 & 0 & 1.119518 & -0.336582 & -0.051751 \\
\hline 3 & 1 & 0 & 2.662937 & -3.366289 & 0.200320 \\
\hline 4 & 6 & 0 & -0.070859 & 0.395306 & -0.644396 \\
\hline 5 & 6 & 0 & 0.545925 & 0.358344 & 2.222268 \\
\hline 6 & 6 & 0 & 1.497530 & 1.980398 & 0.399946 \\
\hline 7 & 1 & 0 & 2.578073 & 0.374969 & 1.437981 \\
\hline 8 & 6 & 0 & 0.558639 & 3.108609 & 0.890143 \\
\hline 9 & 1 & 0 & 0.036748 & 2.953252 & 1.839917 \\
\hline 10 & 1 & 0 & 2.496951 & 2.319599 & 0.125788 \\
\hline 11 & 1 & 0 & -0.415476 & -0.022529 & -1.592029 \\
\hline 12 & 6 & 0 & 0.485674 & 1.845799 & -0.766420 \\
\hline 13 & 6 & 0 & -1.188981 & 0.314536 & 0.411395 \\
\hline 14 & 1 & 0 & 0.817514 & 2.071151 & -1.783341 \\
\hline 15 & 8 & 0 & -0.2 & 2845 & -0.241971 \\
\hline 16 & 1 & 0 & 1.000994 & 4.110646 & 0.875474 \\
\hline 17 & 6 & 0 & -2.607951 & 0.321987 & -0.057740 \\
\hline 18 & 1 & 0 & 2.470023 & -0.413434 & -2.579546 \\
\hline 19 & 1 & 0 & -1.669232 & 0.268789 & 2.460320 \\
\hline 20 & 6 & 0 & 2.493483 & -2.309762 & 0.365282 \\
\hline 21 & 6 & 0 & -0.876152 & 0.313522 & 1.716071 \\
\hline 22 & 1 & 0 & 3.125624 & 1.238972 & -2.020256 \\
\hline 23 & 1 & 0 & 0.824126 & -2.227612 & -0.959696 \\
\hline 24 & 1 & 0 & 6.502292 & -0.756200 & -0.354775 \\
\hline 25 & 1 & 0 & 5.525328 & -1.091728 & 1.092491 \\
\hline 26 & 8 & 0 & 3.985631 & -1.756558 & -0.899692 \\
\hline 27 & 6 & 0 & 3.046515 & 0.168789 & -1.874938 \\
\hline 28 & 1 & 0 & 2.994103 & -1.885111 & 1.226996 \\
\hline 29 & 1 & 0 & 6.356330 & 0.484085 & 0.928056 \\
\hline 30 & 6 & 0 & 5.835670 & -0.311146 & 0.391747 \\
\hline 31 & 6 & 0 & 3.938830 & -0.491764 & -1.051550 \\
\hline 32 & 8 & 0 & 4.714478 & 0.317882 & -0.235644 \\
\hline 33 & 6 & 0 & 1.385761 & -1.684366 & -0.204964 \\
\hline 34 & 1 & 0 & 0.780305 & -0.589875 & 2.726311 \\
\hline 35 & 1 & 0 & 0.655010 & 1.138978 & 2.986938 \\
\hline 36 & 6 & 0 & -5.337719 & 0.451626 & -0.824992 \\
\hline 37 & 6 & 0 & -3.429267 & -0.818207 & -0.098332 \\
\hline 38 & 6 & 0 & -3.203886 & 1.537633 & -0.435337 \\
\hline 39 & 6 & 0 & -4.543719 & 1.602125 & -0.821519 \\
\hline 40 & 6 & 0 & -4.779725 & -0.767320 & -0.450327 \\
\hline 41 & 7 & 0 & -2.882486 & -2.151140 & 0.204078 \\
\hline 42 & 1 & 0 & -2.589885 & 2.431976 & -0.405787 \\
\hline 43 & 1 & 0 & -4.970723 & 2.558216 & -1.109636 \\
\hline 44 & 1 & 0 & -5.363598 & -1.680235 & -0.437659 \\
\hline
\end{tabular}




$\begin{array}{rrrrrr}45 & 1 & 0 & -6.381417 & 0.500528 & -1.118672 \\ 46 & 8 & 0 & -3.607857 & -2.944886 & 0.806482 \\ 47 & 8 & 0 & -1.743782 & -2.405878 & -0.191303\end{array}$

$H F=-1205.4575858$

Table S18 Cartesian coordinates and energy of MPW1K/6-31+G** optimization of transition state from compound 7E to 8out.

Standard orientation:

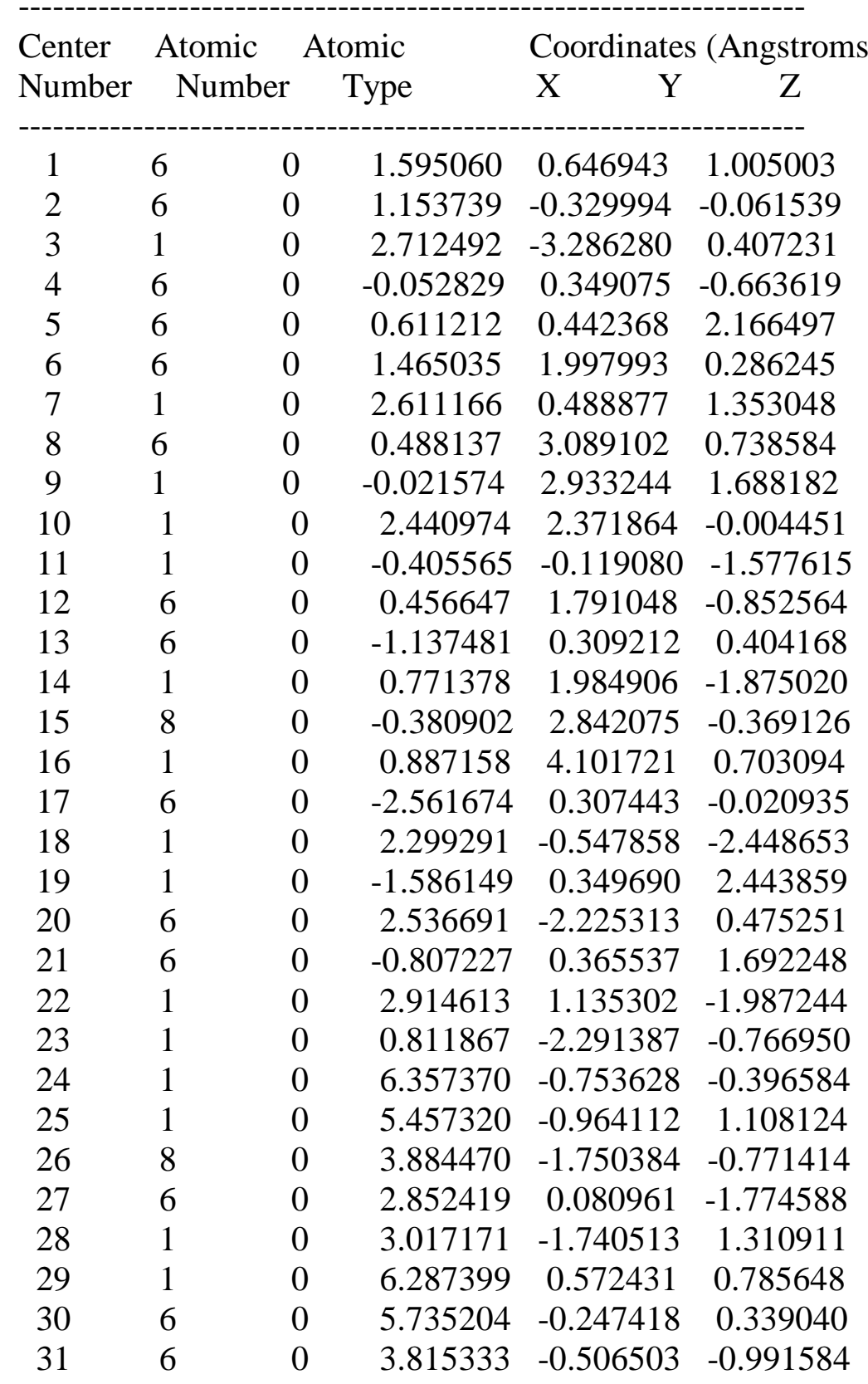




$\begin{array}{rrrrrr}32 & 8 & 0 & 4.605317 & 0.334059 & -0.270706 \\ 33 & 6 & 0 & 1.382785 & -1.686428 & -0.079035 \\ 34 & 1 & 0 & 0.865517 & -0.482869 & 2.688867 \\ 35 & 1 & 0 & 0.719726 & 1.242745 & 2.901330 \\ 36 & 6 & 0 & -5.299397 & 0.418669 & -0.675857 \\ 37 & 6 & 0 & -3.356393 & -0.834861 & -0.091433 \\ 38 & 6 & 0 & -3.188791 & 1.518148 & -0.309514 \\ 39 & 6 & 0 & -4.533052 & 1.573999 & -0.640013 \\ 40 & 6 & 0 & -4.708299 & -0.796507 & -0.389457 \\ 41 & 7 & 0 & -2.781254 & -2.151615 & 0.127860 \\ 42 & 1 & 0 & -2.589008 & 2.414315 & -0.266554 \\ 43 & 1 & 0 & -4.985525 & 2.529298 & -0.860973 \\ 44 & 1 & 0 & -5.268623 & -1.717425 & -0.400765 \\ 45 & 1 & 0 & -6.348310 & 0.461263 & -0.925803 \\ 46 & 8 & 0 & -3.476560 & -2.986599 & 0.660201 \\ 47 & 8 & 0 & -1.652654 & -2.339405 & -0.263465 \\ ----------------------------------------------------------------\end{array}$

Table S19 Energetics of IEFPCM/B3LYP/6-31+G**//B3LYP/6-31+G** $\varepsilon=$ toluene single point calculation of transition state from compound $7 \mathrm{E}$ to 8 out.

Variational PCM results

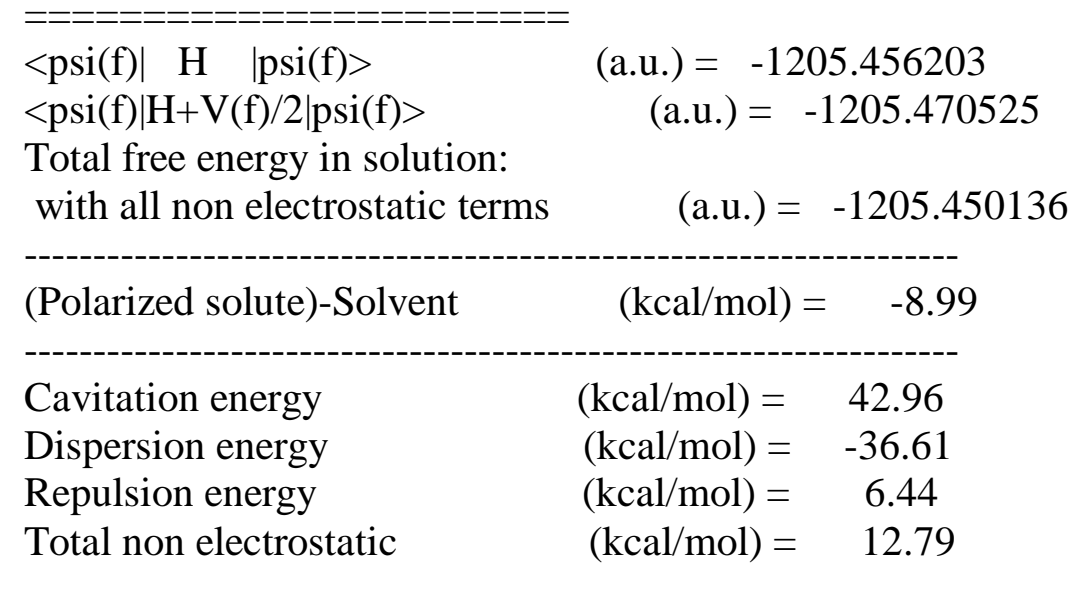

Table S20 Energetics of IEFPCM/B3LYP/6-31+G**//B3LYP/6-31+G** $\varepsilon=$ water single point calculation of transition state from compound $7 \mathrm{E}$ to 8 out.

Variational PCM results

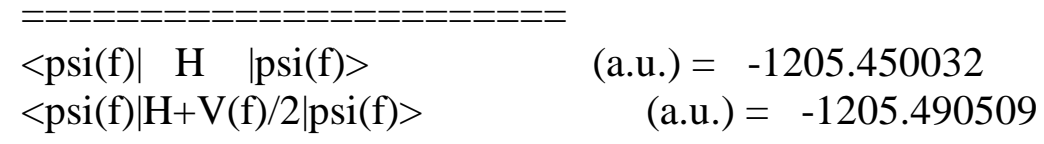


Total free energy in solution:

\begin{tabular}{|c|c|c|}
\hline (Polarized solute)-Solvent & $(\mathrm{kcal} / \mathrm{mol})=$ & -25.40 \\
\hline Cavitation energy & $(\mathrm{kcal} / \mathrm{mol})=$ & 51.34 \\
\hline Dispersion energy & $(\mathrm{kcal} / \mathrm{mol})=$ & -37.86 \\
\hline Repulsion energy & $(\mathrm{kcal} / \mathrm{mol})=$ & 4.84 \\
\hline Total non electrostatic & $(\mathrm{kcal} / \mathrm{mol})=$ & 18.31 \\
\hline
\end{tabular}

Table S21 Energetics of IEFPCM/ MPW1K/6-31+G**//MPW1K/6-31+G** $\varepsilon=$ toluene single point calculation of transition state from compound $7 \mathrm{E}$ to 8out.

Variational PCM results

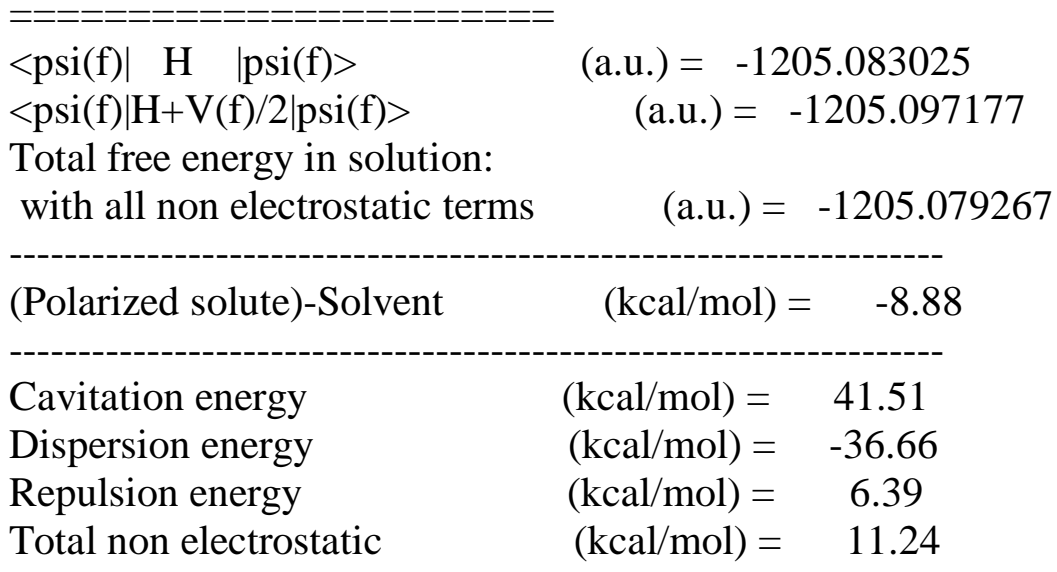

Table S22 Energetics of IEFPCM/ MPW1K/6-31+G**//MPW1K/6-31+G** $\varepsilon=$ water single point calculation of transition state from compound $7 \mathrm{E}$ to 8 out.

Variational PCM results

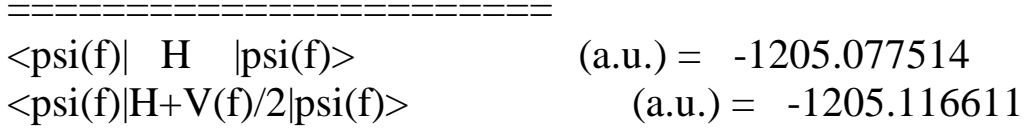

Total free energy in solution:

with all non electrostatic terms

$$
\text { (a.u.) }=-1205.090363
$$

(Polarized solute)-Solvent

$(\mathrm{kcal} / \mathrm{mol})=-24.53$

Cavitation energy

$(\mathrm{kcal} / \mathrm{mol})=49.58$

Dispersion energy

Repulsion energy

$(\mathrm{kcal} / \mathrm{mol})=-37.90$

Total non electrostatic

$(\mathrm{kcal} / \mathrm{mol})=4.79$

$(\mathrm{kcal} / \mathrm{mol})=16.47$ 
Table S23 Cartesian coordinates, energy and imaginary frequency of B3LYP/6-31G* optimization of transition state from compound $7 \mathrm{Z}$ to 8 in.

Standard orientation:

\begin{tabular}{|c|c|c|c|c|c|}
\hline \multirow{2}{*}{$\begin{array}{l}\text { Center } \\
\text { Number }\end{array}$} & \multirow{2}{*}{\multicolumn{2}{|c|}{$\begin{array}{l}\text { Atomic } \\
\text { Number }\end{array}$}} & \multirow{2}{*}{$\begin{array}{r}\text { Atomic } \\
\text { Type }\end{array}$} & \multicolumn{2}{|c|}{ Coordinates (Angstroms } \\
\hline & & & & $X$ & $\mathrm{Z}$ \\
\hline 1 & 6 & 0 & -2.811851 & -0.430457 & 0.972001 \\
\hline 2 & 6 & 0 & -2.120099 & 0.492618 & -0.030261 \\
\hline 3 & 1 & 0 & -2.836844 & 3.002041 & -2.232189 \\
\hline 4 & 6 & 0 & -1.035944 & -0.405597 & -0.628338 \\
\hline 5 & 6 & 0 & -1.877077 & -0.778159 & 2.145165 \\
\hline 6 & 6 & 0 & -3.095767 & -1.621026 & 0.002801 \\
\hline 7 & 1 & 0 & -3.741544 & 0.001889 & 1.354412 \\
\hline 8 & 6 & 0 & -2.562860 & -3.057546 & 0.224870 \\
\hline 9 & 1 & 0 & -2.071285 & -3.262562 & 1.181107 \\
\hline 10 & 1 & 0 & -4.124094 & -1.585007 & -0.363208 \\
\hline 11 & 1 & 0 & -0.496828 & 0.049340 & -1.459143 \\
\hline 12 & 6 & 0 & -1.999624 & -1.552398 & -1.095318 \\
\hline 13 & 6 & 0 & -0.047356 & -0.886202 & 0.440215 \\
\hline 14 & 1 & 0 & -2.313600 & -1.407690 & -2.134832 \\
\hline 15 & 8 & 0 & -1.599190 & -2.918222 & -0.851426 \\
\hline 16 & 1 & 0 & -3.282265 & -3.855784 & 0.006874 \\
\hline 17 & 6 & 0 & 1.334406 & -1.218396 & -0.028815 \\
\hline 18 & 1 & 0 & -1.809282 & 2.634228 & 1.644909 \\
\hline 19 & 1 & 0 & 0.229934 & -1.409991 & 2.457026 \\
\hline 20 & 6 & 0 & -2.237290 & 2.245086 & -1.739169 \\
\hline 21 & 6 & 0 & -0.468214 & -1.071324 & 1.698355 \\
\hline 22 & 1 & 0 & -0.190678 & 1.750427 & 1.918962 \\
\hline 23 & 1 & 0 & -3.769726 & 1.788937 & -0.324044 \\
\hline 24 & 1 & 0 & 1.442471 & 4.149129 & -1.172377 \\
\hline 25 & 1 & 0 & 0.914611 & 2.946941 & -2.373107 \\
\hline 26 & 8 & 0 & -1.161555 & 3.448517 & -0.749920 \\
\hline 27 & 6 & 0 & -0.878259 & 2.251539 & 1.250132 \\
\hline 28 & 1 & 0 & -1.428320 & 1.851042 & -2.345130 \\
\hline 29 & 1 & 0 & 2.446315 & 2.700201 & -1.477732 \\
\hline 30 & 6 & 0 & 1.425705 & 3.080838 & -1.413660 \\
\hline 31 & 6 & 0 & -0.428387 & 2.766482 & 0.052156 \\
\hline 32 & 8 & 0 & 0.806610 & 2.328758 & -0.370296 \\
\hline 33 & 6 & 0 & -2.829297 & 1.442034 & -0.752091 \\
\hline 34 & 1 & 0 & -1.858735 & 0.058556 & 2.858031 \\
\hline 35 & 1 & 0 & -2.282660 & -1.631632 & 2.706543 \\
\hline 36 & 6 & 0 & 3.848465 & -1.905501 & -1.181895 \\
\hline 37 & 6 & 0 & 1.463739 & -2.248928 & -0.980731 \\
\hline
\end{tabular}




$\begin{array}{rrrrrr}38 & 6 & 0 & 2.526123 & -0.543375 & 0.311120 \\ 39 & 6 & 0 & 3.758499 & -0.866779 & -0.264691 \\ 40 & 6 & 0 & 2.691088 & -2.599797 & -1.536661 \\ 41 & 1 & 0 & 0.567569 & -2.796513 & -1.253455 \\ 42 & 7 & 0 & 2.582152 & 0.554923 & 1.290526 \\ 43 & 1 & 0 & 4.631366 & -0.294511 & 0.025958 \\ 44 & 1 & 0 & 2.740745 & -3.418817 & -2.249071 \\ 45 & 1 & 0 & 4.810436 & -2.167581 & -1.612143 \\ 46 & 8 & 0 & 1.891283 & 0.471697 & 2.306072 \\ 47 & 8 & 0 & 3.364271 & 1.476028 & 1.057798\end{array}$

$\mathrm{W}=-435.9947$

$\mathrm{HF}=-1205.3768253$

Sum of electronic and zero-point Energies $=\quad-1204.996923$

Sum of electronic and thermal Energies $=\quad-1204.974942$

Sum of electronic and thermal Enthalpies= $\quad-1204.973998$

Sum of electronic and thermal Free Energies $=\quad-1205.047068$

Table S24 Cartesian coordinates and energy of B3LYP/6-31+G** optimization of transition state from compound $7 \mathrm{Z}$ to 8 in.

Standard orientation:

\begin{tabular}{|c|c|c|c|c|c|}
\hline \multirow{2}{*}{$\begin{array}{l}\text { Center } \\
\text { Number }\end{array}$} & \multirow{2}{*}{\multicolumn{2}{|c|}{$\begin{array}{l}\text { Atomic } \\
\text { Number }\end{array}$}} & \multirow{2}{*}{$\begin{array}{l}\text { Atomic } \\
\text { Type }\end{array}$} & \multicolumn{2}{|c|}{ Coordinates (Angstroms) } \\
\hline & & & & $\mathrm{X}$ & $\mathrm{Z}$ \\
\hline 1 & 6 & 0 & -2.817199 & -0.423566 & 0.978837 \\
\hline 2 & 6 & 0 & -2.134403 & 0.486736 & -0.038478 \\
\hline 3 & 1 & 0 & -2.855143 & 3.018386 & -2.220660 \\
\hline 4 & 6 & 0 & -1.054516 & -0.412543 & -0.639258 \\
\hline 5 & 6 & 0 & -1.868779 & -0.759970 & 2.145076 \\
\hline 6 & 6 & 0 & -3.112363 & -1.623198 & 0.023389 \\
\hline 7 & 1 & 0 & -3.741287 & 0.015149 & 1.366084 \\
\hline 8 & 6 & 0 & -2.584296 & -3.059582 & 0.260098 \\
\hline 9 & 1 & 0 & -2.087319 & -3.256321 & 1.214614 \\
\hline 10 & 1 & 0 & -4.142814 & -1.585860 & -0.335293 \\
\hline 11 & 1 & 0 & -0.529031 & 0.034902 & -1.482326 \\
\hline 12 & 6 & 0 & -2.022333 & -1.567634 & -1.083430 \\
\hline 13 & 6 & 0 & -0.050593 & -0.874291 & 0.425566 \\
\hline 14 & 1 & 0 & -2.340297 & -1.438979 & -2.123053 \\
\hline 15 & 8 & 0 & -1.619918 & -2.932545 & -0.822474 \\
\hline 16 & 1 & 0 & -3.304336 & -3.857250 & 0.047924 \\
\hline
\end{tabular}




$\begin{array}{rrrrrr}17 & 6 & 0 & 1.328589 & -1.202343 & -0.053701 \\ 18 & 1 & 0 & -1.789550 & 2.664026 & 1.650062 \\ 19 & 1 & 0 & 0.241715 & -1.381136 & 2.445368 \\ 20 & 6 & 0 & -2.258623 & 2.249528 & -1.744569 \\ 21 & 6 & 0 & -0.462520 & -1.050681 & 1.689455 \\ 22 & 1 & 0 & -0.172597 & 1.767178 & 1.914213 \\ 23 & 1 & 0 & -3.764235 & 1.812606 & -0.295426 \\ 24 & 1 & 0 & 1.605835 & 4.124414 & -1.081446 \\ 25 & 1 & 0 & 0.868532 & 3.104751 & -2.343186 \\ 26 & 8 & 0 & -1.154244 & 3.472227 & -0.749585 \\ 27 & 6 & 0 & -0.856981 & 2.285566 & 1.255946 \\ 28 & 1 & 0 & -1.448388 & 1.859368 & -2.350306 \\ 29 & 1 & 0 & 2.411817 & 2.608738 & -1.579219 \\ 30 & 6 & 0 & 1.448645 & 3.093506 & -1.415982 \\ 31 & 6 & 0 & -0.418837 & 2.784003 & 0.046947 \\ 32 & 8 & 0 & 0.802671 & 2.325074 & -0.395628 \\ 33 & 6 & 0 & -2.834467 & 1.458183 & -0.738924 \\ 34 & 1 & 0 & -1.845665 & 0.082085 & 2.850914 \\ 35 & 1 & 0 & -2.264284 & -1.609806 & 2.717470 \\ 36 & 6 & 0 & 3.838717 & -1.905873 & -1.219588 \\ 37 & 6 & 0 & 1.443680 & -2.198361 & -1.046163 \\ 38 & 6 & 0 & 2.536084 & -0.568514 & 0.317008 \\ 39 & 6 & 0 & 3.765921 & -0.900418 & -0.263412 \\ 40 & 6 & 0 & 2.665912 & -2.556704 & -1.609972 \\ 41 & 1 & 0 & 0.540401 & -2.719721 & -1.343126 \\ 42 & 7 & 0 & 2.613493 & 0.504990 & 1.325654 \\ 43 & 1 & 0 & 4.650641 & -0.360890 & 0.052697 \\ 44 & 1 & 0 & 2.699710 & -3.348301 & -2.352817 \\ 45 & 1 & 0 & 4.796995 & -2.174568 & -1.652560 \\ 46 & 8 & 0 & 1.910615 & 0.418940 & 2.334043 \\ 47 & 8 & 0 & 3.419004 & 1.414205 & 1.119572 \\ ---------------------------------------------------------\end{array}$

$\mathrm{HF}=-1205.448986$

Table S25 Cartesian coordinates and energy of MPW1K/6-31+G** optimization of transition state from compound $7 \mathrm{Z}$ to 8 in.

Standard orientation:

\begin{tabular}{|c|c|c|c|c|}
\hline \multirow{2}{*}{$\begin{array}{l}\text { Center } \\
\text { Number }\end{array}$} & \multirow{2}{*}{$\begin{array}{l}\text { Atomic } \\
\text { Number }\end{array}$} & \multirow{2}{*}{$\begin{array}{r}\text { Atomic } \\
\text { Type }\end{array}$} & \multicolumn{2}{|c|}{ Coordinates (Angstroms } \\
\hline & & & $X$ & $\mathrm{Z}$ \\
\hline 1 & 6 & -2.7 & -0 . & 0.982219 \\
\hline 2 & 6 & $-2.09 \xi$ & 793 & -0.005274 \\
\hline 3 & 1 & -2.92 & 2.866057 & -2.199869 \\
\hline 4 & 6 & -0.992501 & -0.417298 & -0.612592 \\
\hline
\end{tabular}




\begin{tabular}{|c|c|c|c|c|c|}
\hline 5 & 6 & 0 & -1.803876 & -0.859192 & 2.135066 \\
\hline 6 & 6 & 0 & -2.981943 & -1.698673 & 0.006975 \\
\hline 7 & 1 & 0 & -3.680080 & -0.134363 & 1.369518 \\
\hline 8 & 6 & 0 & -2.388901 & -3.099646 & 0.204835 \\
\hline 9 & 1 & 0 & -1.881349 & -3.289365 & 1.148485 \\
\hline 10 & 1 & 0 & -4.006217 & -1.699693 & -0.352208 \\
\hline 11 & 1 & 0 & -0.473652 & 0.063734 & -1.434761 \\
\hline 12 & 6 & 0 & -1.906504 & -1.576446 & -1.085870 \\
\hline 13 & 6 & 0 & 0.002909 & -0.876586 & 0.435502 \\
\hline 14 & 1 & 0 & -2.232932 & -1.428434 & -2.113333 \\
\hline 15 & 8 & 0 & -1.456175 & -2.908491 & -0.862340 \\
\hline 16 & 1 & 0 & -3.069620 & -3.920866 & -0.014382 \\
\hline 17 & 6 & 0 & 1.385290 & -1.145732 & -0.039262 \\
\hline 18 & 1 & 0 & -1.968605 & 2.406231 & 1.609473 \\
\hline 19 & 1 & 0 & 0.312589 & -1.421793 & 2.426524 \\
\hline 20 & 6 & 0 & -2.290695 & 2.154276 & -1.693015 \\
\hline 21 & 6 & 0 & -0.399982 & -1.097037 & 1.683297 \\
\hline 22 & 1 & 0 & -0.322624 & 1.590257 & 1.862383 \\
\hline 23 & 1 & 0 & -3.835010 & 1.582509 & -0.339571 \\
\hline 24 & 1 & 0 & 1.221309 & 4.213480 & -1.019046 \\
\hline 25 & 1 & 0 & 0.709676 & 3.100337 & -2.296048 \\
\hline 26 & 8 & 0 & -1.355336 & 3.306313 & -0.733231 \\
\hline 27 & 6 & 0 & -1.033704 & 2.061854 & 1.205119 \\
\hline 28 & 1 & 0 & -1.478378 & 1.787661 & -2.304835 \\
\hline 29 & 1 & 0 & 2.255438 & 2.837503 & -1.455489 \\
\hline 30 & 6 & 0 & 1.231521 & 3.175432 & -1.345105 \\
\hline 31 & 6 & 0 & -0.585660 & 2.662956 & 0.057490 \\
\hline 32 & 8 & 0 & 0.655646 & 2.338844 & -0.365152 \\
\hline 33 & 6 & 0 & -2.877402 & 1.292851 & -0.754000 \\
\hline 34 & 1 & 0 & -1.807220 & -0.043099 & 2.861433 \\
\hline 35 & 1 & 0 & -2.176124 & -1.730385 & 2.677855 \\
\hline 36 & 6 & 0 & 3.903259 & -1.724549 & -1.193261 \\
\hline 37 & 6 & 0 & 1.546479 & -2.146866 & -1.001957 \\
\hline 38 & 6 & 0 & 2.542982 & -0.440871 & 0.305214 \\
\hline 39 & 6 & 0 & 3.778472 & -0.710199 & -0.267128 \\
\hline 40 & 6 & 0 & 2.776506 & -2.444913 & -1.558925 \\
\hline 41 & 1 & 0 & 0.671951 & -2.714181 & -1.282555 \\
\hline 42 & 7 & 0 & 2.555180 & 0.639536 & 1.282929 \\
\hline 43 & 1 & 0 & 4.626507 & -0.115653 & 0.031422 \\
\hline 44 & 1 & 0 & 2.852475 & -3.244136 & -2.281240 \\
\hline 45 & 1 & 0 & 4.867593 & -1.947142 & -1.623422 \\
\hline 46 & 8 & 0 & 1.864848 & 0.531167 & 2.269682 \\
\hline 47 & 8 & 0 & 3.297999 & 1.569373 & 1.065191 \\
\hline
\end{tabular}

$\mathrm{HF}=-1205.0781802$ 
Table S26 Energetics of IEFPCM/B3LYP/6-31+G**//B3LYP/6-31+G** $\varepsilon=$ toluene single point calculation of transition state from compound $7 \mathrm{Z}$ to $8 \mathrm{in}$.

Variational PCM results

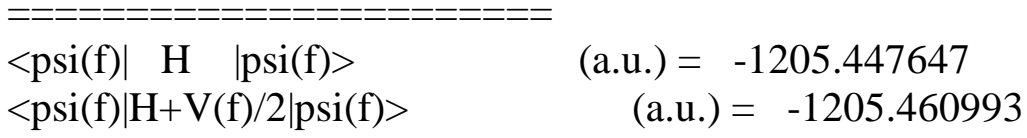

Total free energy in solution:

with all non electrostatic terms $\quad$ (a.u.) $=-1205.440118$

\begin{tabular}{lll} 
(Polarized solute)-Solvent & $(\mathrm{kcal} / \mathrm{mol})=$ & -8.37 \\
\hdashline Cavitation energy & $(\mathrm{kcal} / \mathrm{mol})=$ & 42.14 \\
Dispersion energy & $(\mathrm{kcal} / \mathrm{mol})=$ & -35.06 \\
Repulsion energy & $(\mathrm{kcal} / \mathrm{mol})=$ & 6.02 \\
Total non electrostatic & $(\mathrm{kcal} / \mathrm{mol})=$ & 13.10
\end{tabular}

Table S27 Energetics of IEFPCM/B3LYP/6-31+G**//B3LYP/6-31+G** $\varepsilon=$ water single point calculation of transition state from compound $7 \mathrm{Z}$ to $8 \mathrm{in}$.

Variational PCM results

\begin{tabular}{|c|c|}
\hline $\begin{array}{l}<\operatorname{psi}(\mathrm{f})|\mathrm{H} \quad| \mathrm{psi}(\mathrm{f})> \\
<\operatorname{psi}(\mathrm{f})|\mathrm{H}+\mathrm{V}(\mathrm{f}) / 2| \mathrm{psi}(\mathrm{f})>\end{array}$ & $\begin{array}{l}\text { (a.u.) }=-1205.440699 \\
\quad(\text { a.u. })=-1205.479565\end{array}$ \\
\hline $\begin{array}{l}\text { Total tree energy in solution: } \\
\text { with all non electrostatic terms }\end{array}$ & $($ a.u. $)=-1205.449871$ \\
\hline (Polarized solute)-Solvent & $(\mathrm{kcal} / \mathrm{mol})=$ \\
\hline Cavitation energy & (kcal $/ \mathrm{mol}$ \\
\hline Dispersion energy & $(\mathrm{kcal} / \mathrm{mol})=$ \\
\hline Repulsion energy & $(\mathrm{kcal} / \mathrm{mol})=$ \\
\hline Total non electrostatic & $(\mathrm{kcal} / \mathrm{mol})=$ \\
\hline
\end{tabular}

Table S28 Energetics of IEFPCM/ MPW1K/6-31+G**//MPW1K/6-31+G** $\varepsilon=$ toluene single point calculation of transition state from compound $7 \mathrm{Z}$ to $8 \mathrm{in}$.

Variational PCM results 


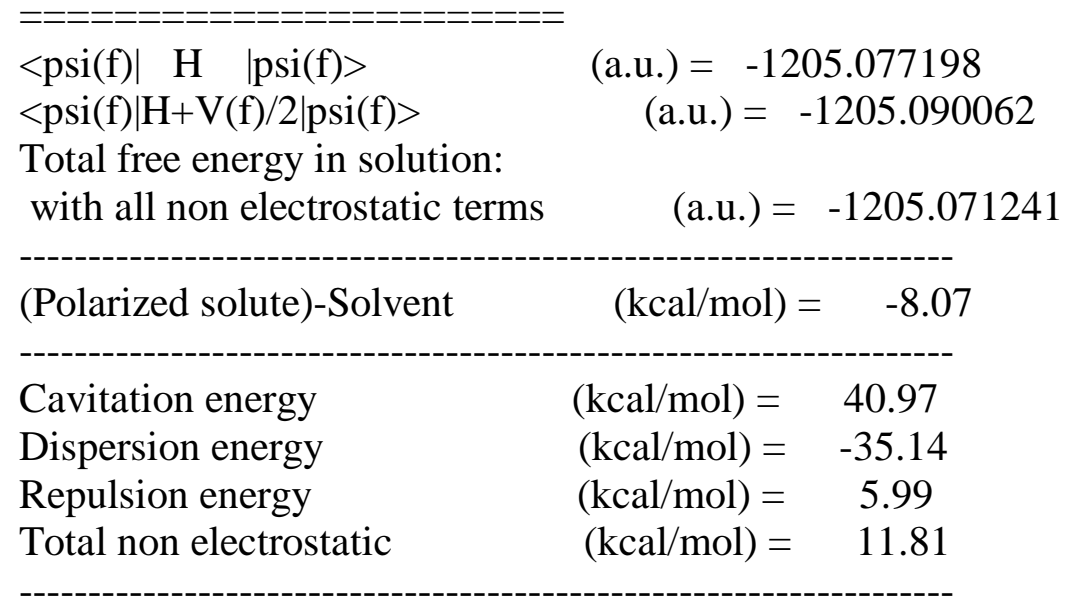

Table S29 Energetics of IEFPCM/ MPW1K/6-31+G**//MPW1K/6-31+G** $\varepsilon=$ water single point calculation of transition state from compound $7 \mathrm{Z}$ to $8 \mathrm{in}$.

Variational PCM results

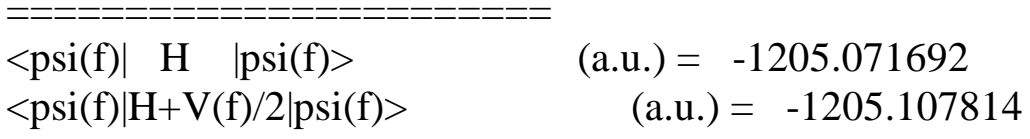

Total free energy in solution:

with all non electrostatic terms

(a.u.) $=-1205.080562$

$($ Polarized solute)-Solvent $\quad(\mathrm{kcal} / \mathrm{mol})=-22.67$

$\begin{array}{lcc}\text { Cavitation energy } & (\mathrm{kcal} / \mathrm{mol})= & 48.93 \\ \text { Dispersion energy } & (\mathrm{kcal} / \mathrm{mol})= & -36.33 \\ \text { Repulsion energy } & (\mathrm{kcal} / \mathrm{mol})= & 4.50 \\ \text { Total non electrostatic } & (\mathrm{kcal} / \mathrm{mol})= & 17.10\end{array}$

Table S30 Cartesian coordinates, energy and imaginary frequency of B3LYP/6-31G* optimization of transition state from compound $7 \mathrm{Z}$ to 8out.

Standard orientation:

\begin{tabular}{|c|c|c|c|c|}
\hline \multirow{2}{*}{$\begin{array}{l}\text { Center } \\
\text { Number }\end{array}$} & \multirow{2}{*}{$\begin{array}{l}\text { Atomic } \\
\text { Number }\end{array}$} & \multirow{2}{*}{$\begin{array}{r}\text { Atomic } \\
\text { Type }\end{array}$} & \multicolumn{2}{|c|}{ Coordinates (Angstroms) } \\
\hline & & & $\mathrm{X}$ & $\mathrm{Z}$ \\
\hline 1 & 6 & -1.486037 & 0.305017 & -0.995230 \\
\hline 2 & 6 & 0.851887 & 0.726352 & -0.388164 \\
\hline 3 & 6 & 0.953498 & 1.480636 & -1.491559 \\
\hline 4 & 6 & -1.682983 & -2.108540 & -1.309079 \\
\hline
\end{tabular}




\begin{tabular}{|c|c|c|c|c|c|}
\hline 5 & 1 & 0 & -0.991443 & -2.359647 & -0.515539 \\
\hline 6 & 1 & 0 & -1.968260 & -2.932781 & -1.951679 \\
\hline 7 & 6 & 0 & -1.776786 & -0.799597 & -1.775367 \\
\hline 8 & 1 & 0 & -2.345010 & -0.625609 & -2.688618 \\
\hline 9 & 6 & 0 & -3.751166 & 0.013079 & 0.244695 \\
\hline 10 & 1 & 0 & -4.262623 & 0.121780 & -0.701505 \\
\hline 11 & 1 & 0 & -1.107915 & 4.463609 & 0.804658 \\
\hline 12 & 8 & 0 & -3.311934 & -2.261081 & -0.151143 \\
\hline 13 & 6 & 0 & -3.356052 & -1.256969 & 0.628569 \\
\hline 14 & 8 & 0 & -2.826313 & -1.355550 & 1.902834 \\
\hline 15 & 1 & 0 & -1.752534 & 1.302450 & 1.793146 \\
\hline 16 & 1 & 0 & -3.871694 & 0.795472 & 0.984114 \\
\hline 17 & 1 & 0 & 1.935966 & 1.727065 & -1.890957 \\
\hline 18 & 6 & 0 & -0.245565 & 2.011302 & -2.240600 \\
\hline 19 & 8 & 0 & -0.191509 & 2.652536 & 1.372469 \\
\hline 20 & 1 & 0 & 0.112473 & 3.847541 & -0.346115 \\
\hline 21 & 1 & 0 & -0.127976 & 3.085125 & -2.442942 \\
\hline 22 & 6 & 0 & 2.060584 & 0.264142 & 0.359561 \\
\hline 23 & 1 & 0 & -2.755824 & 2.841726 & 0.011873 \\
\hline 24 & 1 & 0 & -2.414553 & 1.920786 & -2.151627 \\
\hline 25 & 1 & 0 & -0.493597 & -0.498620 & 0.811046 \\
\hline 26 & 1 & 0 & -0.303719 & 1.525430 & -3.225684 \\
\hline 27 & 6 & 0 & -1.122255 & 1.619674 & 0.959066 \\
\hline 28 & 6 & 0 & -2.594479 & -2.682002 & 2.375614 \\
\hline 29 & 1 & 0 & -1.820700 & -3.194824 & 1.795658 \\
\hline 30 & 1 & 0 & -3.510462 & -3.281920 & 2.337392 \\
\hline 31 & 1 & 0 & -2.265938 & -2.572057 & 3.411346 \\
\hline 32 & 6 & 0 & -1.571965 & 1.746214 & -1.476083 \\
\hline 33 & 6 & 0 & -1.721282 & 2.530059 & -0.143134 \\
\hline 34 & 6 & 0 & -0.679609 & 3.560504 & 0.353424 \\
\hline 35 & 6 & 0 & -0.535551 & 0.397067 & 0.191301 \\
\hline 36 & 6 & 0 & 4.414804 & -0.441773 & 1.777458 \\
\hline 37 & 6 & 0 & 2.926729 & -0.749229 & -0.086587 \\
\hline 38 & 6 & 0 & 2.412568 & 0.905978 & 1.558818 \\
\hline 39 & 6 & 0 & 3.564077 & 0.554069 & 2.262007 \\
\hline 40 & 6 & 0 & 4.099547 & -1.090765 & 0.589129 \\
\hline 41 & 7 & 0 & 2.619928 & -1.539450 & -1.288901 \\
\hline 42 & 1 & 0 & 1.769259 & 1.705409 & 1.912408 \\
\hline 43 & 1 & 0 & 3.804050 & 1.071511 & 3.186821 \\
\hline 44 & 1 & 0 & 4.734681 & -1.866391 & 0.177791 \\
\hline 45 & 1 & 0 & 5.316026 & -0.712855 & 2.319136 \\
\hline 46 & 8 & 0 & 1.439352 & -1.797484 & -1.522536 \\
\hline 47 & 8 & 0 & 3.567969 & -1.925714 & -1.973053 \\
\hline
\end{tabular}

$W=-356.6994$ 
$\mathrm{HF}=-1205.3828456$

Sum of electronic and zero-point Energies $=\quad-1205.003675$

Sum of electronic and thermal Energies $=\quad-1204.981330$

Sum of electronic and thermal Enthalpies $=\quad-1204.980385$

Sum of electronic and thermal Free Energies $=\quad-1205.055613$

Table S31 Cartesian coordinates and energy of B3LYP/6-31+G** optimization of transition state from compound $7 \mathrm{Z}$ to 8out.

Standard orientation:

\begin{tabular}{|c|c|c|c|c|c|}
\hline \multirow{2}{*}{$\begin{array}{l}\text { Center } \\
\text { Number }\end{array}$} & \multirow{2}{*}{$\begin{array}{l}\text { Atomic } \\
\text { Number }\end{array}$} & \multirow{2}{*}{\multicolumn{2}{|c|}{$\begin{array}{l}\text { Atomic } \\
\text { Type }\end{array}$}} & \multicolumn{2}{|c|}{ Coordinates (Angstroms) } \\
\hline & & & & $\mathrm{X}$ & $\mathrm{Z}$ \\
\hline 1 & 6 & 0 & -1.437433 & 0.358816 & -1.008015 \\
\hline 2 & 6 & 0 & 0.881204 & 0.716989 & -0.318920 \\
\hline 3 & 6 & 0 & 1.029137 & 1.503849 & -1.395899 \\
\hline 4 & 6 & 0 & -1.671087 & -2.043274 & -1.408130 \\
\hline 5 & 1 & 0 & -1.026741 & -2.333238 & -0.588680 \\
\hline 6 & 1 & 0 & -1.957058 & -2.838950 & -2.084148 \\
\hline 7 & 6 & 0 & -1.742652 & -0.716003 & -1.822767 \\
\hline 8 & 1 & 0 & -2.281076 & -0.501213 & -2.744659 \\
\hline 9 & 6 & 0 & -3.805467 & 0.049823 & 0.172312 \\
\hline 10 & 1 & 0 & -4.256541 & 0.216446 & -0.795390 \\
\hline 11 & 1 & 0 & -1.071641 & 4.447478 & 0.946274 \\
\hline 12 & 8 & 0 & -3.378523 & -2.214006 & -0.309746 \\
\hline 13 & 6 & 0 & -3.439223 & -1.241567 & 0.510886 \\
\hline 14 & 8 & 0 & -2.966262 & -1.410418 & 1.801095 \\
\hline 15 & 1 & 0 & -1.783311 & 1.270215 & 1.803067 \\
\hline 16 & 1 & 0 & -3.928229 & 0.802924 & 0.940500 \\
\hline 17 & 1 & 0 & 2.027605 & 1.741118 & -1.758805 \\
\hline 18 & 6 & 0 & -0.137380 & 2.084513 & -2.158270 \\
\hline 19 & 8 & 0 & -0.187647 & 2.607729 & 1.473426 \\
\hline 20 & 1 & 0 & 0.180116 & 3.863178 & -0.189446 \\
\hline 21 & 1 & 0 & 0.004614 & 3.162137 & -2.315730 \\
\hline 22 & 6 & 0 & 2.055619 & 0.191842 & 0.437811 \\
\hline 23 & 1 & 0 & -2.709692 & 2.875328 & 0.039822 \\
\hline 24 & 1 & 0 & -2.310441 & 2.019684 & -2.139061 \\
\hline 25 & 1 & 0 & -0.517890 & -0.521358 & 0.799624 \\
\hline 26 & 1 & 0 & -0.172774 & 1.636853 & -3.161682 \\
\hline 27 & 6 & 0 & -1.123032 & 1.602231 & 0.999362 \\
\hline 28 & 6 & 0 & -2.822935 & -2.761779 & 2.249337 \\
\hline 29 & 1 & 0 & -2.067162 & -3.302634 & 1.672742 \\
\hline
\end{tabular}




$\begin{array}{rrrrrr}30 & 1 & 0 & -3.773318 & -3.300812 & 2.174697 \\ 31 & 1 & 0 & -2.514500 & -2.692435 & 3.293804 \\ 32 & 6 & 0 & -1.491903 & 1.814418 & -1.443575 \\ 33 & 6 & 0 & -1.674126 & 2.557118 & -0.091093 \\ 34 & 6 & 0 & -0.638003 & 3.560273 & 0.471876 \\ 35 & 6 & 0 & -0.528822 & 0.396054 & 0.211244 \\ 36 & 6 & 0 & 4.357734 & -0.641559 & 1.876312 \\ 37 & 6 & 0 & 2.961707 & -0.750968 & -0.078109 \\ 38 & 6 & 0 & 2.338431 & 0.693655 & 1.720665 \\ 39 & 6 & 0 & 3.464245 & 0.278427 & 2.432927 \\ 40 & 6 & 0 & 4.111560 & -1.152304 & 0.605048 \\ 41 & 7 & 0 & 2.713501 & -1.407869 & -1.373391 \\ 42 & 1 & 0 & 1.672540 & 1.445611 & 2.131279 \\ 43 & 1 & 0 & 3.651404 & 0.688293 & 3.421035 \\ 44 & 1 & 0 & 4.781509 & -1.866419 & 0.140210 \\ 45 & 1 & 0 & 5.237944 & -0.960069 & 2.425605 \\ 46 & 8 & 0 & 1.566156 & -1.784364 & -1.613881 \\ 47 & 8 & 0 & 3.678110 & -1.573988 & -2.122810 \\ -------------------------------------------------------------------\end{array}$

$\mathrm{HF}=-1205.456591$

Table S32 Cartesian coordinates and energy of MPW1K/6-31+G** optimization of transition state from compound $7 \mathrm{Z}$ to 8out.

Standard orientation:

\begin{tabular}{|c|c|c|c|c|}
\hline \multirow{2}{*}{$\begin{array}{l}\text { Center } \\
\text { Number }\end{array}$} & \multirow{2}{*}{$\begin{array}{l}\text { Atomic } \\
\text { Number }\end{array}$} & \multirow{2}{*}{$\begin{array}{r}\text { Atomic } \\
\text { Type }\end{array}$} & \multicolumn{2}{|c|}{ Coordinates (Angstroms } \\
\hline & & & $\mathrm{X}$ & $\mathrm{Z}$ \\
\hline 1 & 6 & -1.492857 & 0.294911 & -0.956309 \\
\hline 2 & 6 & 0.820151 & 0.694383 & -0.353343 \\
\hline 3 & 6 & 0.934551 & 1.417989 & -1.464811 \\
\hline 4 & 6 & -1.677708 & -2.092303 & -1.248287 \\
\hline 5 & 1 & -0.995399 & -2.328379 & -0.448550 \\
\hline 6 & 1 & -1.933079 & -2.923107 & -1.884957 \\
\hline 7 & 6 & -1.727650 & -0.800796 & -1.754383 \\
\hline 8 & 1 & -2.262405 & -0.636328 & -2.681028 \\
\hline 9 & 6 & -3.618234 & 0.069923 & 0.170258 \\
\hline 10 & 1 & -4.115656 & 0.176645 & -0.776745 \\
\hline 11 & 1 & -0.994599 & 4.433025 & 0.759731 \\
\hline 12 & 8 & -3.244406 & -2.200094 & -0.190457 \\
\hline 13 & 6 & -3.296521 & -1.201384 & 0.583907 \\
\hline 14 & 8 & -2.826688 & -1.323658 & 1.852160 \\
\hline 15 & 1 & -1.749520 & 1.334798 & 1.799712 \\
\hline 16 & 1 & -3.739948 & 0.852265 & 0.900710 \\
\hline
\end{tabular}




\begin{tabular}{rrrrrr}
17 & 1 & 0 & 1.917789 & 1.638913 & -1.860675 \\
18 & 6 & 0 & -0.247081 & 1.947633 & -2.216159 \\
19 & 8 & 0 & -0.166808 & 2.617642 & 1.360813 \\
20 & 1 & 0 & 0.194320 & 3.739442 & -0.365558 \\
21 & 1 & 0 & -0.111744 & 3.008407 & -2.437228 \\
22 & 6 & 0 & 2.017111 & 0.231002 & 0.389834 \\
23 & 1 & 0 & -2.694076 & 2.871855 & 0.011696 \\
24 & 1 & 0 & -2.398937 & 1.898723 & -2.114184 \\
25 & 1 & 0 & -0.519807 & -0.493301 & 0.853540 \\
26 & 1 & 0 & -0.312440 & 1.443611 & -3.183374 \\
27 & 6 & 0 & -1.111808 & 1.621181 & 0.968316 \\
28 & 6 & 0 & -2.674161 & -2.638997 & 2.336523 \\
29 & 1 & 0 & -1.904859 & -3.182972 & 1.794047 \\
30 & 1 & 0 & -3.608394 & -3.191843 & 2.261746 \\
31 & 1 & 0 & -2.386446 & -2.540017 & 3.377525 \\
32 & 6 & 0 & -1.557313 & 1.722168 & -1.448189 \\
33 & 6 & 0 & -1.678616 & 2.520100 & -0.140352 \\
34 & 6 & 0 & -0.606789 & 3.508628 & 0.334279 \\
35 & 6 & 0 & -0.555024 & 0.391225 & 0.226610 \\
36 & 6 & 0 & 4.365056 & -0.483083 & 1.771717 \\
37 & 6 & 0 & 2.896074 & -0.737280 & -0.088501 \\
38 & 6 & 0 & 2.348710 & 0.823488 & 1.607693 \\
39 & 6 & 0 & 3.497964 & 0.466099 & 2.292949 \\
40 & 6 & 0 & 4.067550 & -1.083473 & 0.563705 \\
41 & 7 & 0 & 2.594254 & -1.479638 & -1.303049 \\
42 & 1 & 0 & 1.697204 & 1.596409 & 1.986760 \\
43 & 1 & 0 & 3.725062 & 0.945614 & 3.233478 \\
44 & 1 & 0 & 4.715769 & -1.824253 & 0.123429 \\
45 & 1 & 0 & 5.265590 & -0.755203 & 2.300421 \\
46 & 8 & 0 & 1.457077 & -1.858245 & -1.457563 \\
47 & 8 & 0 & 3.509745 & -1.702560 & -2.062688 \\
-------------------------------------------------------------- \\
\hline 3
\end{tabular}

$\mathrm{HF}=-1205.0837049$

Table S33 Energetics of IEFPCM/B3LYP/6-31+G**//B3LYP/6-31+G** $\varepsilon=$ toluene single point calculation of transition state from compound $7 \mathrm{Z}$ to 8 out.

Variational PCM results

\begin{tabular}{|c|c|}
\hline $\begin{array}{l}<\operatorname{psi}(\mathrm{f})|\mathrm{H} \quad| \operatorname{psi}(\mathrm{f})> \\
<\operatorname{psi}(\mathrm{f})|\mathrm{H}+\mathrm{V}(\mathrm{f}) / 2| \operatorname{psi}(\mathrm{f})>\end{array}$ & $\begin{array}{l}\text { (a.u.) }=-1205.455468 \\
\quad(\text { a.u. })=-1205.469837\end{array}$ \\
\hline $\begin{array}{l}\text { Total free energy in solution: } \\
\text { with all non electrostatic terms }\end{array}$ & $($ a.u. $)=-1205$. \\
\hline
\end{tabular}




\begin{tabular}{lll} 
(Polarized solute)-Solvent & $(\mathrm{kcal} / \mathrm{mol})=$ & -9.02 \\
\hdashline Cavitation energy & $(\mathrm{kcal} / \mathrm{mol})=$ & 42.95 \\
Dispersion energy & $(\mathrm{kcal} / \mathrm{mol})=$ & -36.11 \\
Repulsion energy & $(\mathrm{kcal} / \mathrm{mol})=$ & 6.37 \\
Total non electrostatic & $(\mathrm{kcal} / \mathrm{mol})=$ & 13.21
\end{tabular}

Table S34 Energetics of IEFPCM/B3LYP/6-31+G**//B3LYP/6-31+G** $\varepsilon=$ water single point calculation of transition state from compound $7 \mathrm{Z}$ to 8 out.

Variational PCM results

\begin{tabular}{|c|c|c|}
\hline $\begin{array}{l}<p s i(f)|\quad H \quad| p s i(f)> \\
<p s i(f)|H+V(f) / 2| p s i(f)>\end{array}$ & $\begin{array}{l}\text { (a.u.) }=-120 \\
\quad(\text { a.u. })=-1\end{array}$ & $\begin{array}{l}5.449299 \\
1205.489902\end{array}$ \\
\hline $\begin{array}{l}\text { Total free energy in solution: } \\
\text { with all non electrostatic terms }\end{array}$ & (a.u.) $=$ & -1205.460023 \\
\hline (Polarized solute)-Solvent & $(\mathrm{kcal} / \mathrm{mol})=$ & -25.48 \\
\hline Cavitation energy & $(\mathrm{kcal} / \mathrm{mol})=$ & 51.32 \\
\hline Dispersion energy & $(\mathrm{kcal} / \mathrm{mol})=$ & -37.36 \\
\hline Repulsion energy & $(\mathrm{kcal} / \mathrm{mol})=$ & 4.79 \\
\hline Total non electrostatic & $(\mathrm{kcal} / \mathrm{mol})=$ & 18.75 \\
\hline
\end{tabular}

Table S35 Energetics of IEFPCM/ MPW1K/6-31+G**//MPW1K/6-31+G** $\varepsilon=$ toluene single point calculation of transition state from compound $7 \mathrm{Z}$ to 8 out.

Variational PCM results

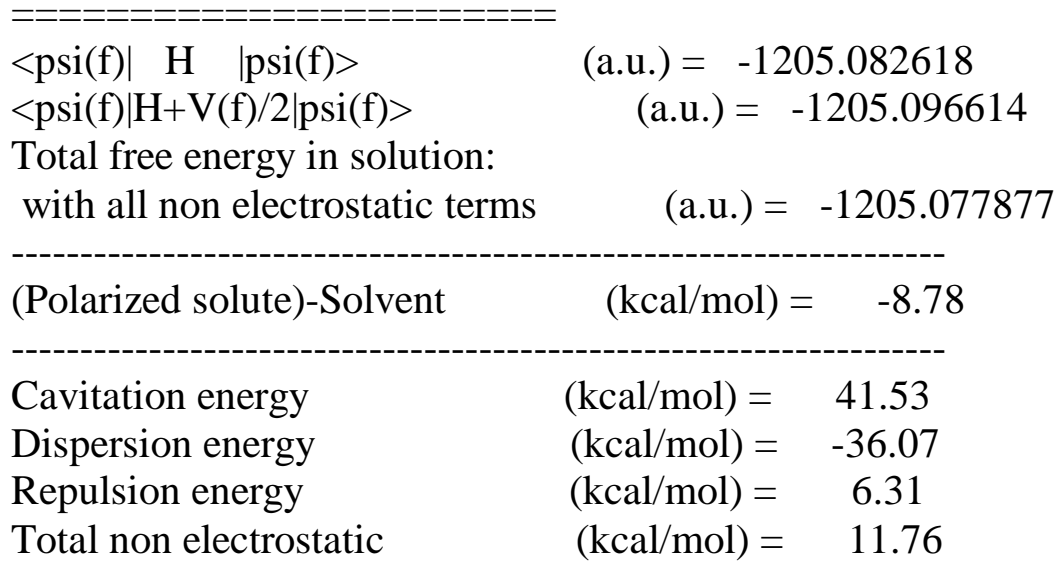


Table S36 Energetics of IEFPCM/ MPW1K/6-31+G**//MPW1K/6-31+G** $\varepsilon=$ water single point calculation of transition state from compound $7 \mathrm{Z}$ to 8out.

Variational PCM results

\begin{tabular}{|c|c|}
\hline $\begin{array}{l}\langle\mathrm{psi}(\mathrm{f})|\mathrm{H} \quad| \mathrm{psi}(\mathrm{f})\rangle \\
\langle\mathrm{psi}(\mathrm{f})|\mathrm{H}+\mathrm{V}(\mathrm{f}) / 2| \mathrm{psi}(\mathrm{f})\rangle \\
\text { Total free energy in solution: } \\
\text { with all non electrostatic terms }\end{array}$ & $\begin{array}{l}(\text { a.u. })=-1205.077190 \\
\quad(\text { a.u. })=-1205.116115\end{array}$ \\
\hline (Polarized solute)-Solvent & $(\mathrm{kcal} / \mathrm{mol})=$ \\
\hline Cavit & $(\mathrm{kcal} / \mathrm{mol})=$ \\
\hline Dispersion energy & $(\mathrm{kcal} / \mathrm{mol})=-37.31$ \\
\hline Repulsion energy & $(\mathrm{kcal} / \mathrm{mol})=$ \\
\hline Total non electrostatic & $(\mathrm{kcal} / \mathrm{mol})=$ \\
\hline
\end{tabular}

